\title{
El cuestionario Five to Fifteen (FTF) para una Evaluación Integral del Desarrollo: Propiedades Psicométricas y Caracterización de Muestra de Niños Chilenos The Five to Fifteen Questionnaire (FTF) for a Comprehensive Assessment of Development: Psychometric Properties and Characterization of a Sample of Chilean Children
}

\author{
María Fernanda Beltrán-Ortiz \\ Universidad de Talca, Chile \\ Helena Todd de Barra \\ Patricio Franzani \\ Cristian Martinich \\ Corporación para el Desarrollo del Aprendizaje (CDA), Chile \\ Ramón D. Castillo \\ Universidad de Talca, Chile
}

(Rec: 06 de octubre de 2011 / Acep: 11 de mayo de 2012)

\begin{abstract}
Resumen
El impacto de los trastornos del desarrollo, puede ser mitigado con un diagnóstico temprano. El cuestionario Five to Fifteen (FTF) podría ser una adecuada herramienta de diagnóstico precoz para estos trastornos. Son descritas las propiedades psicométricas, y la caracterización de 322 niños chilenos de desarrollo típico, entre 5 y 15 años de edad.

La consistencia interna y la estabilidad temporal fluctuaron entre. 83 y .93 y entre .44 y .86 , respectivamente. Del AFE emergieron 4 dimensiones que explicaron el $65.4 \%$ de la varianza $\left(\mathrm{KMO}=.93 ; \chi^{2}=3.113,77 ; g l=\right.$ $231 ; p<.01)$, predominando un factor de desarrollo general.

Fueron encontradas diferencias por género y edad. El desempeño de los niños chilenos y suecos fue similar a los 5 años. Pero desde los 6 años en adelante fueron observadas diferencias en casi la totalidad de los dominios. Nosotros concluimos que el FTF es válido y confiable midiendo el desarrollo infantil.

Palabras clave: Cuestionario Five to Fifteen (FTF) - desarrollo infantil.
\end{abstract}

\begin{abstract}
The impact of developmental disorders can be mitigated if early diagnoses are implemented. The Five to Fifteen questionnaire (FTF) could be a suitable tool for early diagnosis of these disorders.

We describe the psychometric properties and the characterization of 322 Chilean children of typical development, between 5 and 15 years of age.

Levels of consistency and temporal stabilitywere fluctuating between .83 and .93 , and between .44 and .86, respectively. From an Exploratory Factorial Analysis emerge 4 dimensions that account for $65.4 \%$ of variance $\left(\mathrm{KMO}=.93 ; \chi^{2}=3,113.77 ; g l=231 ; p<.01\right)$, where a general development dimension is predominating. Differences were found by gender and age. Performance of Chilean and Sweden children indicates similarities at 5 years of age. However from 6 years of age onwards differences were found in almost all domains. We conclude that FTF is avalid and reliable instrument measuring children's development.

Keywords: Five to Fifteen (FTF) Questionnaire - child development.
\end{abstract}

Agradecimientos: El equipo de investigación siente una especial gratitud a los directores, profesores, padres y apoderados de las escuelas y colegios de Talca, por su colaboración en este proyecto, especialmente a Ximena Rudloff.

Los autores agradecen por sus comentarios y sugerencias a Felipe Cornejo, Ricardo Rey Andrés Fresno, académicos de la Facultad de Psicología de la Universidad de Talca.

En memoria de nuestra querida colega y amiga Helena Todd de Barra, quien falleció en Santiago de Chile, el 10 de septiembre del 2011.

Correspondencia: Ramón D. Castillo, Facultad de Psicología, Universidad de Talca, Avenida Lircay s/n, Talca, Chile. Teléfono (5671) 201567, Fax (5671)

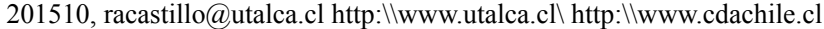




\section{Introducción}

La salud infantil es tema crucial para la psicología clínica, la pediatría y las disciplinas relacionadas. Especialmente por la creciente evidencia científica de la relación existente entre retrasos del desarrollo, problemas de salud mental y otros problemas sociales (Bedregal, Scharager, Breinbahuer, Solari y Molina, 2007; Leslie, Hurlburt, Landsverk, Barth y Slymen, 2004). Una serie de estudios longitudinales han identificado que los problemas emocionales y conductuales tempranos, constituyen un predictor de disfunciones posteriores, y que ciertas conductas desadaptativas tempranas tienden a consolidarse con los años, pudiendo conducir a distintos trastornos psiquiátricos y problemas de salud mental (Babinsky, Hartsough y Lambert, 1999; De la Barra, Toledo y Rodríguez, 2003).

La prevalencia global de los trastornos del desarrollo infantil se ha estimado entre un $16 \%$ a un $18 \%$ de los niños, de los cuales casi el $90 \%$ se relaciona a problemas de aprendizaje, lenguaje o retardo mental. La cifra de prevalencia aumentaría a un $22 \%$, de incluirse los problemas del comportamiento (Avaria, 2005). Y aun cuando la prevalencia de estos trastornos afecta a 1 de cada 5 niños, es importante tomar en cuenta que los trastornos del desarrollo de los primeros años, tenderán a remitir en función tanto de las pautas educativas como de los modelos de evaluación y tratamiento ofrecidos por el medio social (Bedregal, 2008). Es por este motivo, que adecuados procesos de diagnóstico e intervención temprana, pueden prevenir o reducir el impacto de los trastornos en el individuo, su familia y los sistemas de salud (Belfer, 2008; De la Barra et al., 2003; Schonaut, Maggiolo, Barbieri, Rojas y Salgado, 2007).

\section{Instrumentos de evaluación del desarrollo infantil}

La evaluación tradicional del desarrollo consiste en la comparación entre las características actuales de un área o áreas del desarrollo del niño con normas externas preestablecidas (González, 2007). Existen diversas metodologías de evaluación del desarrollo y sus trastornos, que difieren en cuanto a su utilidad y alcance. Por ejemplo, el desarrollo infantil puede ser evaluado directamente en el niño a través de exámenes físicos, entrevistas, baterías de evaluación de habilidades, test proyectivos, cuestionarios de auto-informe; o bien indirectamente por medio de informantes que interactúan de manera permanente con el niño, como es el caso de padres y profesores (Amador, Forns, Guárdia y Peró, 2006; Bedregal, 2008; Ferrán, Claustre, Canals, Esparó, Bellespi y Doménech, 2008; Haeussler y Marchant, 2002; Wechsler, 1999).

Si bien algunos síntomas de trastornos del desarrollo pueden limitarse a un solo contexto, otros requieren para el diagnóstico, su presencia en más de un contexto (American Psychiatric Association, 2002). Así entonces, la información que proporcionan padres y profesores es necesaria en el proceso diagnóstico (Amador et al., 2006; Cova, Maganto y Melipillán, 2005; Ferrán et al., 2008).

Investigaciones han demostrado que la información suministrada por padres y profesores permite diagnosticar con una precisión elevada algunos trastornos del desarrollo, destacando que no existen diferencias significativas en la valoración que ambos informantes realizan del desarrollo del niño y de trastornos específicos como el TDAH (Amador, Forns y Martorell, 2001; Kadesjö, Janols, Korkman, Mickelsson, Strand, Trillingsgaard, y Gillberg, 2004; Kordas, Stoltzfus, López, Alatorre y Rosado, 2005; Power, Doherthy, Panichelli, Karustis, Eiraldi, Anastopoulus y Du Paul, 1998). Sin embargo, investigaciones más recientes indican que los padres son capaces de percibir de mejor manera los comportamientos internalizados de sus hijos y al evaluar con mayor severidad sus síntomas, logran mayor concordancia con otros criterios de evaluación (Amador et al., 2001; Cova et al., 2005; Ferrán, et al., 2008).

En Chile existen variados instrumentos de evaluación del desarrollo, algunos son de carácter global, mientras que otros están orientados a aspectos específicos. Sin embargo, son pocos los que están validados, y de ellos, en solo dos casos su aplicación está establecida a través de políticas de gobierno. En este sentido, el Ministerio de Salud establece que la Escala de Evaluación del Desarrollo Psicomotor (EEDP) construida por Rodríguez, Arancibia y Undurraga (1976) y el Test de desarrollo psicomotor (Tepsi), desarrollado por Haeusler y Marchant (2002), son los instrumentos que se aplican en el marco de los programas de estimulación y evaluación del desarrollo psicomotor y Chile Crece Contigo (Bedregal, 2008). El primero evalúa las áreas motora gruesa y fina, coordinación, lenguaje y social en niños de 0 a 2 años, mientras que el segundo evalúa las áreas de motricidad, coordinación y lenguaje en niños de 0 a 5 años.

Estas pruebas establecidas por las políticas de gobierno, si bien aportan información relevante acerca del desarrollo infantil, están relegadas a la evaluación de áreas específicas y orientadas a rangos de edad limitados. Además, evalúan el desarrollo del niño a través de la observación directa, sin tomar en cuenta la información que los padres pueden aportar en relación al comportamiento y las pautas de interacción de sus hijos.

En Chile hay disponible en el mercado una serie de instrumentos validados, que utilizan la valoración de los padres para evaluar el desarrollo de sus hijos y detectar algunas patologías asociadas. Entre estas escalas se encuentra:

El Inventario de problemas conductuales y emocionales (IPCS), que es un instrumento de evaluación desarrollado y validado en Chile por Rodríguez, Lira, Arancibia y Bralic (1996), orientado a detectar problemas conductuales, socioemocionales, evaluar problemas maternos y situaciones estresantes, en niños de 3 a 5 años.

La Lista de Chequeo del comportamiento del niño (Child Behavior Checklist, CBCL) es un instrumento desarrollado por 
Achenbach, que evalúa competencia social y los problemas conductuales-emocionales en niños (Bohlin y Janols, 2004; Leslie et al., 2004). Ha sido validado al contexto chileno en niños de 6 a 11 años de edad (Montenegro, Bralic, Edwards, Izquierdo y Maltes, 1983, en Cova, et al., 2005).

La lista de chequeo, denominada Pediatric Symptom Checklist, es una escala desarrollada por Jellineck, que evalúa riesgos conductuales como déficit de atención e hiperactividad, trastornos de conducta, trastornos depresivos y trastornos de ansiedad, en niños de 6 a 16 años. En Chile el estudio de validación de la escala ha sido realizado solo con niños de primero y sexto año básico (De la Barra, Toledo y Rodríguez, 2002; George, Siraquian y Mores, 1995).

En general todas las escalas revisadas se encuentran orientadas a la evaluación de ciertas áreas del desarrollo, lo que no permite obtener una visión global del estado real del niño. Por otra parte, algunos instrumentos, como es el caso de las escalas IPCS y Pediatric Symptom Checklist, están dirigidos a un rango etario reducido; en el primer caso por su estructura y en el segundo caso porque su validación en Chile solo ha sido realizada en primero y sexto año de enseñanza básica.

Es por estas limitaciones que emerge la necesidad de contar con un instrumento que evalúe el proceso del desarrollo desde un punto de vista global, tomando en cuenta todas sus áreas, en un rango amplio de edad. Así, en el año 2007 comenzaron a estudiarse en Chile las propiedades psicométricas del cuestionario Five to Fifteen (González, 2007), cuyas características permitirían superar algunas de las limitaciones que presentan los instrumentos actualmente en uso en Chile.

\section{El Cuestionario Five to Fifteen (FTF)}

El Cuestionario Five to Fifteen para padres, fue desarrollado en 1997 por Lars-Olof Jands, Marit Korkman, Bjôrn Kadesjo, Gerd Strand, Katarina Mickelsson y Anegen Trillingsgaard. Todos ellos investigadores clínicos pertenecientes al denominado grupo de consenso Nórdico en Síndrome de Disfunción Cerebral Mínima (MBD), Déficit Atencional, de Control motor y Percepción (DAMP) y Déficit atencional con desorden de hiperactividad (ADHD). Grupo que tiene una amplia experiencia acerca del trastorno de déficit atencional por hiperactividad (TDAH) y sus problemas asociados (Kadesjö et al., 2004).

El objetivo original fue desarrollar una entrevista clínica para padres que generara suficiente información para complementar el diagnóstico de TDAH y sus patologías comorbidas, en vista que el diseño de una entrevista clínica lo suficientemente comprensiva y que no ocupara demasiado tiempo fue muy difícil; un cuestionario para padres fue un objetivo más realista (Bohlin y Janols, 2004; Kadesjö et al., 2004).

La idea fue que el instrumento fuese contestado por los padres, previo a la entrevista clínica, recogiendo información global del desarrollo del niño, así como de los comportamientos típicos para desórdenes psiquiátricos comunes en la infancia, evitando la extensión innecesaria de la entrevista con los padres y optimizando así los tiempos para una intervención (Kadesjö et al., 2004; Korkman, Jaakkola, Ahlroth, Pesonen y Turunen, 2004; Korkman, Kadesjö, Trillingsgaard, Mickelson, Janols, Strand y Gillberg, 2005).

Complementariamente, una vez puntuadas y tabuladas las respuestas, este cuestionario es una herramienta de utilidad para el clínico porque ayuda a clarificar en qué forma un determinado deterioro puede afectar las habilidades del niño con las demandas de la vida diaria; y posibilita para discutir con los padres acerca de los problemas del desarrollo del niño y dar orientación acerca de posibles estrategias de acción (González, 2007; Korkman et al., 2004; Korkman et al., 2005).

El cuestionario está dirigido a niños de 5 a 15 años (condición que da origen a su nombre "five to fifteen"). Posee 181 ítems relativos a habilidades y conductas del niño en diferentes áreas del desarrollo, cada uno de los cuales contiene una declaración que expresa dificultades del niño en ciertas tareas que los padres deben valorar, realizando una comparación entre sus hijos y niños de la misma edad (Airaksinen, Mickelsson y Jokela, 2004; Bohlin y Janols, 2004; Kadesjö et al., 2004; Korkman et al., 2004; Trillingsgaard, Damm, Sommer, Jepsen, Ostergaard, Frydenberg y Thomsen, 2004).

Los ítems se agrupan en 8 áreas o dominios del desarrollo: Funciones Motoras (FM), Funciones Ejecutivas (FE), Percepción (P), Memoria (M), Lenguaje (L), Aprendizaje (A), Habilidades Sociales (HS) y Problemas EmocionalesConductuales (PE/C), los que, exceptuando Memoria y Habilidades sociales, se dividen en 20 subdominios. En los subdominios de Atención e Hiperactividad-Impulsividad se incluyen preguntas semejantes a los síntomas correspondientes a criterios diagnósticos de TDAH, contenidos en el manual DSM IV (Airaksinen et al., 2004; Kadesjö et al., 2004; Korkman et al., 2004). Cada ítem tiene tres opciones de respuesta, calificadas de 0 a 2 puntos, dependiendo del grado de deterioro de cada función. Además se incluyen preguntas abiertas que permiten una descripción de problemas específicos y ejemplos de dificultades en variados ámbitos. También tiene una aproximación a las fortalezas del niño y otros aspectos positivos (Airaksinen et al., 2004; Kadesjö et al., 2004; Korkman et al., 2004).

La combinación de información cuantitativa y cualitativa permite una descripción comprensiva del estado cognitivo y comportamental del niño, lo que aporta al diagnóstico, permite conocer las peculiaridades de cada caso y con esto contrastar diagnósticos (Korkman et al., 2004).

Las propiedades psicométricas de este instrumento han sido ampliamente estudiadas en investigaciones realizadas en los países europeos nórdicos; y hay una investigación 
realizada en Chile con padres de niños que cursan Kínder y Primer año de enseñanza básica (González, 2007).

\section{Confiabilidad del Cuestionario FTF}

Los dominios y subdominios del FTF han demostrado tener buenos niveles de confiabilidad. Los niveles de consistencia interna del cuestionario han fluctuado desde .86 para el dominio de Funciones motoras a .96 para el dominio de Aprendizaje, y desde .69 para el subdominio de Percepción visual, hasta .94 para el subdominio de Habilidades sociales (Kadesjöet al., 2004). En un estudio realizado con una muestra normal de 471 niños de 6 a 8 años, se reportaron coeficientes de confiabilidad desde .84 a .99 para todos los dominios (Airaksinen et al., 2004). En otra investigación, en la que fueron utilizadas solo 5 escalas del FTF, los niveles de confiabilidad fluctuaron de .78 para Habilidades motoras finas, .79 para Percepción, .85 para Memoria, y .89 para las escalas de Atención e impulsividad y Lenguaje (Korkman et al., 2004). En otra investigación con una muestra conformada por niños con TDAH y otros trastornos asociados, se obtuvieron índices de confiabilidad que oscilaron entre .84 y .94 para los ocho dominios (Trillingsgaard et al., 2004).

En Chile se realizó una investigación con dos muestras de niños pertenecientes a Kínder y Primer año básico. En una primera muestra se aplicó la escala a 541 padres de niños, obteniéndose un índice de consistencia interna de .98 para la escala, en ambos niveles de escolaridad. Para los diferentes dominios, los índices de consistencia interna fluctuaron entre .77 para Percepción y .92 para Problemas emocionales-conductuales en Kínder; y entre .81 para Funciones motoras a .93 para Aprendizaje en Primero básico (González, 2007). Posteriormente, en una segunda muestra de 170 niños de la ciudad de Talca, se obtuvieron índices de consistencia interna de la escala que fluctuaron entre .95 y .96 para Kínder y Primer año básico respectivamente, mientras que los dominios presentaron índices que fueron desde .63 para Percepción a .88 para Problemas emocionales-conductuales en Kínder; y desde .69 para Percepción a .91 para Problemas emocionales-conductuales, Funciones Ejecutivas y Habilidades Sociales en Primer año básico (González, 2007).

En cuanto al acuerdo interjuez, en un estudio realizado por Kadesjö et al. (2004) se analizaron los niveles de acuerdo entre padres, encontrando ausencia de diferencias significativas entre las medias de respuestas de ambos, con correlaciones de Pearson que oscilaron de .67 para Lenguaje a .65 para Percepción, lo que da cuenta de buenos niveles de acuerdo entre padres. La confiabilidad test-retest fue analizada con 6 a 8 semanas de diferencia en una muestra de 100 sujetos. En el caso de los Dominios del FTF fueron obtenidas correlaciones de Pearson entre ambas mediciones que oscilaron entre .74 para Memoria y .91 para Funciones ejecutivas; y desde .55 a .89 para los subdominios de Comunicación y Percepción temporal, respectivamente.

\section{Validez de criterio del cuestionario FTF}

En cuanto a la validez de criterio del FTF es posible afirmar que este no solo se relaciona con escalas que miden ajuste social, sino que además se correlaciona con escalas de inteligencia y de funcionamiento neuropsicológico. Por ejemplo (Bohlin y Janols, 2004), en una muestra de 1.500 niños suizos, fueron comparadas las puntuaciones obtenidas en el cuestionario FTF con los resultados obtenidos de la Lista de Chequeo del Comportamiento del Niño (CBCL), desarrollada por Achenbach. Al comparar las escalas equivalentes de ambos cuestionarios, fueron obtenidos coeficientes de correlación que oscilaron entre 0.17 y 0.82 , todos estadísticamente significativos. En esta misma línea, en un estudio conducido por Trillingsgaard et al. (2004), el cual utilizó como criterio las puntuaciones del WISC III, determinó que el índice de comprensión verbal y el coeficiente de inteligencia verbal de la escala WISC III correlacionaban significativamente con el dominio de Lenguaje del FTF (0.37 y 0.43 respectivamente). Correlaciones más bajas, pero significativas, fueron encontradas entre el dominio de Percepción del FTF y el índice de organización perceptual del WISC III (0.23).

Korkman et al. (2004), relacionó las puntaciones del FTF con la escala NEPSY en un grupo de niños con riesgo neuropsicológico. Encontrándose correlaciones significativas entre los dominios que estos cuestionarios tienen en común, tales como: el subdominio de Habilidades motoras finas con el área Sensorio-motora (0.44); el subdominio de Atención e impulsividad con el área de Atención (0.21); el subdominio de Percepción con el área Viso-espacial (0.43); y el dominio de Memoria con el área Memoria y aprendizaje (0.32) y con el área de Lenguaje (0.40).

\section{Validez predictiva del cuestionario FTF}

La evidencia acerca de la capacidad para predecir la membrecía de los niños a un determinado grupo, o detectar la presencia-ausencia de una determinada condición (niños con trastornos del desarrollo versus niños con desarrollo típico), es heterogénea, ya que el único estudio apuntado a este tipo de validez indica que el cuestionario FTF tiene una muy buena capacidad para detectar a los niños con problemas en su desarrollo, pero no clasifica correctamente a los sujetos sanos.

Korkman et al. (2004), administraron el cuestionario a una muestra de 769 niños. Los niños con puntuaciones superiores fueron asignados al grupo de riesgo de tener desórdenes del desarrollo, mientras que el grupo de control fue formado al azar con una muestra de niños que no superaron el punto de corte en ningún dominio (percentil 90). A continuación ambos grupos fueron sometidos a una evaluación con el NEPSY, encontrándose que los participantes pertenecientes 
al grupo de riesgo obtuvieron desempeños significativamente más pobres en esta escala. En cuanto a la sensibilidad y especificidad, un $93 \%$ de los niños diagnosticados en riesgo con el FTF presentaron también debilidades significativas en el NEPSY, encontrándose que el cuestionario FTF posee una buena sensibilidad. Sin embargo, el FTF presentó una gran cantidad de falsos positivos (63\%), lo que da cuenta de una baja especificidad.

\section{Validez de constructo del cuestionario FTF}

Los constructos explorados apuntan a la existencia de varias dimensiones subyacentes, las cuales dependen de si el grupo evaluado está constituido por niños con alteraciones del desarrollo o niños normales. En el estudio realizado por Bohlin y Janols (2004) se analizó la dimensionalidad del cuestionario FTF, por medio de un análisis de componentes principales con rotación oblicua. Se seleccionaron dos factores, identificados como dificultades de aprendizaje y problemas socioemocionales, en donde las escalas de Percepción corporal y Habilidades motoras gruesas no tuvieron ninguna carga relevante en estos factores.

En un estudio similar, efectuado con niños diagnosticados con TDAH, Bruce, Thernlund y Nettelbladt (2006) realizaron un análisis de componentes principales con rotación Varimax, que arrojó 6 factores, que reflejaron síntomas de problemas clínicos y explicaron un $73 \%$ de la varianza. Estos factores fueron denominados: Habilidades cognitivas; Habilidades motoras y percepción; Emoción, socialización y conducta; Atención; Habilidades literarias; y control.

En el estudio realizado en Chile por González (2007), la validez de constructo fue estimada por medio de un análisis de factorización de ejes principales, con rotación Oblimin, en dos muestras de niños chilenos. Obteniéndose en la primera muestra dos dimensiones. La primera de ellas agrupó funciones cognitivas y la segunda agrupó a las funciones socioafectivas y de ajuste, mientras que la escala de Lenguaje quedó fuera de ambas dimensiones. En una segunda muestra, el mismo análisis arrojó una sola dimensión que explicó el $55.86 \%$ de la variabilidad de los datos y agrupó a todas las escalas del cuestionario FTF.

\section{El Cuestionario FTF y las diferencias por género y edad}

Los estudios reportan diferencias significativas entre las puntuaciones obtenidas por hombres y mujeres, aun cuando existen divergencias sobre la magnitud de estas diferencias $\mathrm{y}$ los dominios involucrados (Airaksinen et al., 2004; Bohlin y Janols, 2004; Bruce et al., 2006; Kadesjö et al., 2004).

Algunos estudios han revelado que los niños muestran un deterioro significativamente mayor al de las niñas (Airaksinen, et al., 2004; Bohlin y Janols, 2004; Bruce et al., 2006; Kadesjö et al., 2004). Las mayores diferencias fueron en los dominios de Funciones ejecutivas y
Habilidades motoras (Kadesjö et al., 2004); Percepción y Habilidades motoras (Bohlin y Janols, 2004); Habilidades motoras y Aprendizaje (Bruce et al., 2006); y Habilidades sociales (Airaksinen et al., 2004). En el estudio realizado en Chile, fueron detectadas diferencias significativas en las áreas de Habilidades sociales y Funciones ejecutivas, donde estas las niñas muestran un mejor desempeño que los niños (González, 2007). En contraste con lo anterior, en un estudio realizado con una muestra clínica de TDAH se encontró que las niñas presentan deterioros más severos que los niños en los dominios de Lenguaje y Habilidades sociales (Trillingsgaard et al., 2004).

En general, las investigaciones concuerdan en señalar que la edad de los niños está asociada directamente con las puntuaciones obtenidas en el cuestionario FTF. Sin embargo, existen diferencias en cuanto a los dominios implicados. Por ejemplo, Kadesjö et al. (2004), detectó diferencias significativas en los puntajes del FTF, de acuerdo a la edad, de manera que los niños pequeños presentan más problemas que los adolescentes y preadolescentes, en todos los dominios, exceptuando los de Habilidades sociales y Problemas emocionales-conductuales.

Otros estudios similares muestran una disminución de los deterioros en el desarrollo, a medida que se avanza en edad, en los dominios de Habilidades motoras y Percepción (Bohlin y Janols, 2004), Lenguaje (Trillingsgaard et al., 2004); Percepción, Memoria y Lenguaje (Airaksinen et al., 2004), mientras que el efecto inverso se observa en los dominios de Atención e Hiperactividad (Bohlin y Janols, 2004).

En Chile, González (2007) encontró que los alumnos de primer año básico presentaban desempeños significativamente superiores a los de alumnos de Kínder, en las áreas de Funciones Motoras, Percepción, Memoria, Lenguaje, Funciones Ejecutivas y Aprendizaje. La conclusión preliminar estableció que el FTF fue capaz de detectar las mejoras en el desempeño, entre el paso de la etapa preescolar al primer año de la etapa escolar.

\section{Cuestionario FTF y educación de los padres}

En las investigaciones realizadas hasta la fecha, existen evidencias divergentes en relación a la influencia que el nivel educacional de los padres ejerce sobre el desarrollo del niño. En este sentido, algunos estudios indican que la educación de los padres no se asocia a los resultados obtenidos en todos los dominios del cuestionario FTF (Airaksinen et al., 2004; Korkman et al., 2004). Sin embargo, otros estudios indican que padres con algún grado de formación académica, reportan una cantidad significativamente menor de problemas del desarrollo en sus hijos, en todos los dominios, a excepción de Funciones motoras y Memoria (Bohlin y Janols, 2004; Kadesjö et al., 2004).

En síntesis, existe una alta prevalencia de los trastornos del desarrollo infantil. Es asumido que este tipo de trastornos 
tenderá a remitir en función de diagnósticos e intervenciones oportunas y tempranas. Por esto es necesario contar con instrumentos que permitan evaluar el desarrollo de manera global e integral, con el fin de generar diagnósticos e intervenciones que logren prevenir los efectos que estos trastornos ocasionan en el individuo, su familia y los sistemas de salud (Bedregal, 2008; Belfer, 2008; De la Barra, Toledo y Rodríguez, 2003; Schonaut, et al, 2007).

En Chile existen múltiples instrumentos que evalúan áreas del desarrollo y sus trastornos asociados, sin embargo presentan limitaciones tales como los rangos de edad reducidos o bien que están orientados a un aspecto del desarrollo, sin considerar que el desarrollo es un proceso global y multidimensional.

En este contexto, el cuestionario FTF surge como una herramienta cuyas ventajas se asocian a la diversidad de áreas del desarrollo que evalúa; al amplio rango de edad al que está dirigido (de cinco a 15 años); y a la capacidad para detectar precozmente trastornos como el Déficit Atencional con Hiperactividad (TDAH) y sus síntomas comórbidos.

Tendiente a validar este instrumento en el contexto chileno, como una herramienta de evaluación del desarrollo y detección de TDAH, el año 2007 comenzaron estudios pilotos destinados a evaluar las propiedades psicométricas de la escala FTF en una muestra de niños chilenos, con desarrollo típico, provenientes de Kínder y Primer año de enseñanza básica, encontrándose índices de confiabilidad y validez adecuados (González, 2007). En base a esos resultados, se decidió investigar sus propiedades en una muestra más diversa, que contemplara todo el rango etario para el cual fue diseñado el instrumento, analizando en esta oportunidad la estabilidad temporal de los puntajes por medio de la confiabilidad test-retest, determinando su estructura factorial, analizando el comportamiento de las puntuaciones del FTF según género y escolaridad, para finalmente comparar el desempeño de la muestra de niños chilenos con una muestra de niños suecos.

Fue hipotetizado que los índices de consistencia y la estabilidad temporal del cuestionario FTF, deberían ser al menos similares a los obtenidos en las investigaciones previamente realizadas con este instrumento. Respecto a la validez, fue anticipada una estructura factorial de una dimensión general del desarrollo y al menos cinco dimensiones específicas. En cuanto a la validez discriminante, fue anticipado que los niños deberían presentar mayores dificultades que las niñas, y que la presencia de problemas disminuyera al aumentar la edad o los niveles de escolaridad.

\section{Método}

\section{Participantes}

En diciembre del año 2008 se entregaron 603 cuestionarios, de los cuales solo un $53 \%$ fue devuelto, por lo que la muestra definitiva quedó compuesta por 322 sujetos (ver Tabla 1). De estos, 179 (55.6\%) niñas y 143 niños (44.4\%), que cursaban entre Kínder y Primer año de enseñanza media, provenientes de colegios municipalizados y particular subvencionados de la ciudad de Talca, los cuales fueron seleccionados a través de un muestreo no probabilístico por disponibilidad.

Para estudiar específicamente la confiabilidad por estabilidad temporal, en septiembre del año 2009 se distribuyeron 85 cuestionarios a padres que completaron la escala previamente, de los cuales solo un $64.7 \%$ fue devuelto. Así entonces, la muestra definitiva para esta fase quedó compuesta por 55 sujetos. De estos, 29 (52.7\%) niñas y 26 $(47.3 \%)$ niños, que cursaban entre $1^{\circ}$ y $7^{\circ}$ básico.

\section{Instrumentos}

El cuestionario Five to fifteen (FTF) fue traducido en Chile al español por Helena Todd de Barra y Juan Mallea (González, 2007). Es un instrumento desarrollado en 1997 por Lars-Olof Jands, Marit Korkman, Bjôrn Kadesjo, Gerd Strand, Katarina Mickelsson y Anegen Trillingsgaard, todos investigadores clínicos con vasta experiencia en trastorno de déficit atencional por hiperactividad (TDAH) y sus problemas asociados (Kadesjö et al., 2004).

La escala, dirigida a padres y cuidadores de niños de entre 5 y 15 años, está compuesta por 181 ítems que evalúan 8 dominios: Funciones motoras, Funciones ejecutivas, Percepción, Memoria, Lenguaje, Aprendizaje, Habilidades sociales y Problemas emocionales-conductuales. Los que, a

Tabla 1. Distribución de la muestra según género y curso.

\begin{tabular}{|c|c|c|c|c|c|c|c|c|c|c|c|}
\hline \multirow[t]{2}{*}{ Recuento } & \multicolumn{10}{|c|}{ Escolaridad } & \multirow[t]{2}{*}{ Total } \\
\hline & Kínder & $1^{\circ} \mathrm{EB}$ & $2^{\circ} \mathrm{EB}$ & $3^{\circ} \mathrm{EB}$ & $4^{\circ} \mathrm{EB}$ & $5^{\circ} \mathrm{EB}$ & $6^{\circ} \mathrm{EB}$ & $7^{\circ} \mathrm{EB}$ & $8^{\circ} \mathrm{EB}$ & $1^{\circ} \mathrm{EM}$ & \\
\hline Niños & 19 & 14 & 17 & 12 & 19 & 19 & 35 & 14 & 23 & 7 & 179 \\
\hline Niñas & 29 & 17 & 18 & 5 & 23 & 16 & 15 & 10 & 8 & 2 & 143 \\
\hline Total & 48 & 31 & 35 & 17 & 42 & 35 & 50 & 24 & 31 & 9 & 322 \\
\hline
\end{tabular}

Nota. La correlación entre la edad y la escolaridad fue de $.97(p<.01)$, motivo por el cual se asume que son indicadores análogos. EB: Enseñanza Básica. EM: Enseñanza Media. 
excepción de Memoria y Aprendizaje, se dividen a su vez en subdominios (Tabla 2).

El cuestionario tiene tres opciones de respuesta, a las cuales se les asigna cierta puntuación: $0=$ "no aplica"; $1=$ "aplica algunas veces/en cierto grado"; 2 = "definitivamente aplica". Los resultados son expresados como puntajes de dominios o de subdominios. Estas puntuaciones (0-2) se obtienen promediando el puntaje obtenido en todos los ítems administrados, correspondientes al dominio o subdominio. Bajas puntuaciones indican escasas dificultades reportadas por los padres, mientras que puntajes altos son indicadores de mayores dificultades reportadas por los padres en las diferentes áreas (Airaksinen, et al., 2004; Kadesjö et al., 2004; Korkman, et al., 2005; Trillingsgaard et al., 2004).

\section{Procedimiento}

Se estableció contacto con el director o jefe de la unidad técnico pedagógica de los establecimientos. Una vez contactados los profesores de cada curso, se acordó la fecha, hora y modalidad de la aplicación. Para la aplicación del FTF se utilizaron dos procedimientos, en el primero se asistió a la reunión de apoderados para informarles a los padres sobre los objetivos e implicancias de la investigación, presentes en una carta de consentimiento informado, mientras que en la segunda modalidad se envió esta información por medio de la libreta de comunicaciones del alumno.

En ambos casos, el cuestionario fue entregado para ser contestado en casa y enviado de vuelta al colegio por medio de los alumnos. Para el análisis de la confiabilidad por estabilidad temporal, fueron seleccionados 85 cuestionarios cuya tasa de omisión fue menor a seis preguntas y se envió un nuevo cuestionario, por medio de la libreta de comunicaciones, a partir del noveno mes de haber realizado la primera medición.

Requerimientos éticos: En esta investigación se contó con la autorización y el apoyo de los directores de los establecimientos educacionales involucrados. A los participantes de este estudio (padres de los niños) se les entregó un consentimiento informado, en el que se detallaron los objetivos y características del proyecto, se explicitó el

Tabla 2. Dominios y subdominios del cuestionario FTF.

\begin{tabular}{|c|c|c|c|}
\hline Dominios & $\mathrm{N}^{\circ}$ de preguntas & Subdominios & $\begin{array}{l}\mathrm{N}^{\circ} \mathrm{d} \mathrm{e} \\
\text { Ítems }\end{array}$ \\
\hline \multirow[t]{2}{*}{ 1. Funciones Motoras (FM) } & $1-17$ & Funciones motoras gruesas & 7 \\
\hline & & Funciones motoras finas & 10 \\
\hline \multirow[t]{4}{*}{ 2. Funciones Ejecutivas (FE) } & $18-42$ & Atención & 9 \\
\hline & & Hiperactividad/impulsividad & 9 \\
\hline & & Hipoactividad & 4 \\
\hline & & Planificación/Organización & 3 \\
\hline \multirow[t]{4}{*}{ 3. Percepción (P) } & $43-60$ & Percepción espacial & 5 \\
\hline & & Percepción temporal & 4 \\
\hline & & Percepción corporal & 5 \\
\hline & & Percepción visual & 4 \\
\hline 4. Memoria (M) & $61-71$ & Memoria & 11 \\
\hline \multirow[t]{3}{*}{ 5. Lenguaje (L) } & $72-92$ & Comprensión del lenguaje & 5 \\
\hline & & Expresióndel lenguaje & 13 \\
\hline & & Habilidades de comunicación & 3 \\
\hline \multirow[t]{4}{*}{ 6. Aprendizaje (A) } & $93-121$ & Lectoescritura* & 8 \\
\hline & & Matemáticas* & 5 \\
\hline & & Adquisición de conocimientos & 6 \\
\hline & & Situaciones de aprendizaje & 10 \\
\hline 7. Habilidades Sociales (HS) & $122-147$ & Habilidades sociales & 27 \\
\hline \multirow[t]{3}{*}{ 8. Problemas Emocionales / Conductuales (PEC) } & $148-181$ & Problemas psíquicos internalizados & 12 \\
\hline & & Problemas psíquicos Externalizados & 13 \\
\hline & & Obsesivos-compulsivos & 8 \\
\hline
\end{tabular}

Nota. * Estos subdominios no son evaluados en preescolares (Airaksinen, Mickelsson y Jokela, 2004). 
carácter confidencial del manejo de los datos y se solicitó la firma que permitiera utilizar los datos para ser analizados de manera grupal, nunca individualmente. De esta forma, los padres completaron la escala de manera voluntaria.

\section{Resultados}

Los cuestionarios fueron tabulados y los puntajes fueron analizados con técnicas descriptivas univariadas y multivariadas. Fue estimada la confiabilidad (por consistencia interna y estabilidad temporal) y la validez, además de analizar las puntuaciones en función de la edad y el género de los niños. Los puntajes de esta muestra con los puntajes de uno de los países en los cuales se desarrollaron los estudios de validación.

\section{Confiabilidad}

La consistencia interna fue estimada con el coeficiente Alfa de Cronbach (ver Tabla 3). El cuestionario total arrojó un coeficiente de 0.98 . Para los dominios del cuestionario, los índices variaron desde 0.83 para Percepción hasta 0.93 para Aprendizaje. Lo anterior indica que los ítems que integran los dominios del cuestionario están altamente correlacionados entre sí. En cuanto a los subdominios del cuestionario FTF, los índices de consistencia interna oscilaron entre 0.51 para Adquisición de conocimientos y 0.89 para Situaciones de aprendizaje.

Después de un período de al menos nueve meses entre la primera y la segunda aplicación, se analizó la confiabilidad por estabilidad temporal, calculándose el coeficiente de Spearman-Brown (ver Tabla 3). Para todos los dominios se obtuvieron coeficientes estadísticamente significativos entre

Tabla 3. Media, Desviación Estándar y Confiabilidad para Dominios y Subdominios.

\begin{tabular}{|c|c|c|c|c|}
\hline Dominios y subdominios. & Media & $\mathrm{DE}$ & $\begin{array}{c}\text { Coeficiente } \\
\text { Alfa de Cronbach }\end{array}$ & $\begin{array}{c}\text { Coeficiente } \\
\text { Spearman-Brown }\end{array}$ \\
\hline 1. Funciones motoras (FM) & 0.23 & 0.27 & .85 & $.86 * *$ \\
\hline Funciones motoras gruesas & 0.20 & 0.31 & .79 & $.87^{* *}$ \\
\hline Funciones motoras finas & 0.26 & 0.30 & .77 & $.76 * *$ \\
\hline 2. Funciones Ejecutivas (FE) & 0.57 & 0.40 & .93 & $.78^{* *}$ \\
\hline Atención & 0.67 & 0.47 & .88 & $.86^{* *}$ \\
\hline Hiperactividad/impulsividad & 0.55 & 0.50 & .88 & $.69 * *$ \\
\hline Hipoactividad & 0.47 & 0.47 & .70 & $.28 \mathrm{~ns}$ \\
\hline Planificación/Organización & 0.48 & 0.45 & .59 & $.65 * *$ \\
\hline 3. Percepción (P) & 0.29 & 0.28 & .83 & $.50 *$ \\
\hline Percepciónespacial & 0.23 & 0.30 & .55 & $.69^{* *}$ \\
\hline Percepción temporal & 0.51 & 0.53 & .73 & $.64^{* *}$ \\
\hline Percepción corporal & 0.31 & 0.36 & .58 & $.50 *$ \\
\hline Percepción visual & 0.15 & 0.27 & .63 & $.28 \mathrm{~ns}$ \\
\hline 4. Memoria (M) & 0.33 & 0.31 & .83 & $.44 *$ \\
\hline 5. Lenguaje (L) & 0.28 & 0.30 & .91 & $.61 * *$ \\
\hline Comprensión del lenguaje & 0.39 & 0.40 & .77 & $.63^{* *}$ \\
\hline Expresión del lenguaje & 0.26 & 0.32 & .87 & $.58 * *$ \\
\hline Habilidades de comunicación & 0.25 & 0.39 & .75 & $.51 *$ \\
\hline 6. Aprendizaje (A) & 0.55 & 0.40 & .93 & $.54 *$ \\
\hline Lectoescritura & 0.52 & 0.47 & .85 & $.66^{* *}$ \\
\hline Matemáticas & 0.55 & 0.56 & .89 & $.53 *$ \\
\hline Adquisición de conocimientos & 0.54 & 0.35 & .51 & $.46^{*}$ \\
\hline Situaciones de aprendizaje & 0.59 & 0.50 & .89 & $.63 * *$ \\
\hline 7. Habilidades Sociales (HS) & 0.25 & 0.27 & .91 & $.69 * *$ \\
\hline 8. ProblemasEmocionales / Conductuales (PE/C) & 0.29 & 0.29 & .93 & $.73 * *$ \\
\hline Problemas psíquicos internalizados & 0.27 & 0.32 & .84 & $.49^{* *}$ \\
\hline Problemas psíquicos externalizados & 0.40 & 0.36 & .87 & $.82 * *$ \\
\hline Obsesivos-compulsivos & 0.19 & 0.31 & .82 & $.53 * *$ \\
\hline
\end{tabular}

Nota. Los coeficientes Spearman-Brown fueron estimados a partir de las correlaciones de Pearson, donde $* * p<.01$. * $p<.05$. 
ambas aplicaciones, que variaron entre 0.44 para Memoria y 0.86 para Funciones motoras. En relación a los subdominios, la mayoría muestra correlaciones significativas, a excepción de Hipoactividad y Percepción visual, ambas con un valor 0.28 , siendo el subdominio de Funciones motoras gruesas el que obtiene el coeficiente más alto: 0.87 .

\section{Validez de constructo}

Para evaluar la dimensionalidad del cuestionario, se realizó un análisis factorial exploratorio (AFE) de extracción de componentes principales con rotación Oblimin, que da cuenta de un adecuado ajuste del modelo $(K M O=.93 ; \chi 2=$ $3.113,77 ; g l=231 ; p<.01)$. A partir del AFE se detectaron 4 factores (ver tabla 4) que en conjunto, antes de la rotación, explicaron un $65.43 \%$ de la varianza.

El primer factor explicó un $46.23 \%$ de la varianza y fue denominado desarrollo general, porque la cargas altas estuvieron asociadas a todos los subdominios del FTF. El segundo factor, que explicó un $7.32 \%$ de la varianza, fue denominado problemas socioemocionales y de control, porque las cargas más altas mostraron un predominio de los subdominios: Hiperactividad-Impulsividad; Problemas psíquicos internalizados; Problemas psíquicos externalizados; Obsesivos-Compulsivos; y Habilidades Sociales. El tercer factor, explicó un $7.07 \%$ de la varianza y fue denominado cognición - motricidad y lenguaje, porque agrupó a todos los subdominios relacionados con Percepción y Funciones motoras, además cargaron en él los subdominios de Memoria y Expresión del lenguaje. El cuarto factor explicó solo un $4.81 \%$ de la varianza y fue denominado comunicación y aprendizaje escolar, dado que las cargas más altas predominaron en los subdominios de habilidades de Comunicación, Lectoescritura, Expresión del lenguaje, Comprensión del lenguaje, Aplicación y adquisición de destrezas, Memoria y Matemáticas.

\section{Análisis según género y escolaridad}

Fue realizado un análisis de varianza para cada uno de los dominios del cuestionario, en donde variables género y escolaridad actuaron como factores principales. Para los dominios de Funciones Motoras (FM), Memoria (M), Problemas Emocionales-Conductuales (PE-C) y Habilidades Sociales (HS), no fueron observados efectos estadísticamente significativos asociados a las variables género y escolaridad, y tampoco fue significativa su interacción (ver Tabla 5).

Sin embargo, para el dominio de Funciones Ejecutivas (FE) fueron detectadas diferencias significativas por escolaridad, $F(9,283)=1.94 ; p<.05, \eta p 2=.06$, además de un efecto de interacción entre esta variable y el género, $F(9,283)=2.87 ; p<.005 ; \eta p 2=.08$. Las comparaciones múltiples de medias con la prueba de Bonferroni indicaron que los alumnos de Quinto básico tuvieron un puntaje más bajo que los alumnos de Kínder, Segundo, Tercero y Cuarto básico $(p s<.05)$. También fue observado que los alumnos
Tabla 4. Matriz estructural del cuestionario FTF.

\begin{tabular}{|c|c|c|c|c|}
\hline \multirow[b]{2}{*}{ Subdominios } & \multicolumn{4}{|c|}{ Componentes } \\
\hline & 1 & 2 & 3 & 4 \\
\hline Atención & .876 & .417 & .511 & .468 \\
\hline Situaciones de aprendizaje & .831 & .456 & & .539 \\
\hline Planificación/Organización & .764 & .446 & .469 & \\
\hline Hipoactividad & .691 & .579 & .476 & .463 \\
\hline Matemáticas & .682 & & & .605 \\
\hline Hiperactividad/impulsividad & .593 & .559 & .477 & \\
\hline $\begin{array}{l}\text { Problemas psíquicos } \\
\text { externalizados }\end{array}$ & .411 & .862 & & \\
\hline Obsesivos-compulsivos & & .844 & & \\
\hline Habilidades sociales & .456 & .811 & & \\
\hline $\begin{array}{l}\text { Problemas psíquicos } \\
\text { internalizados }\end{array}$ & & .774 & & .442 \\
\hline Percepción del espacio & & & .789 & \\
\hline Habilidades motoras finas & .582 & & .742 & \\
\hline Percepción corporal & & .418 & .742 & \\
\hline Habilidades motoras gruesas & & .495 & .718 & \\
\hline Percepción del tiempo & .560 & & .690 & \\
\hline Memoria & .564 & .414 & .681 & .661 \\
\hline Percepción visual & & & .659 & .523 \\
\hline Habilidades de comunicación & & .483 & & .761 \\
\hline Lectoescritura & .632 & & & .757 \\
\hline Expresión del lenguaje & & .515 & .553 & .723 \\
\hline Comprensión del lenguaje & .606 & .439 & .529 & .721 \\
\hline $\begin{array}{l}\text { Adquisición y aplicación de } \\
\text { destrezas }\end{array}$ & .437 & & & 692 \\
\hline
\end{tabular}

Nota. Este matriz estructural describe la carga de los 22 subdominios del FTF. Considerando que los dominios Memoria (M) y Habilidades Sociales (HS) no están segmentados en subdominios, fueron incluidos en su totalidad. Los componentes transformados a puntajes obtuvieron correlaciones de Pearson altamente significativas, que fluctuaron entre -0.42 y $0.45(p s<.01)$.

de Primero de Enseñanza Media obtuvieron menos puntaje, por lo tanto menores dificultades que los de Kínder, Segundo, Tercero y Cuarto básico $(p s<.05)$ (ver Tabla 5). En relación al efecto de interacción, se pudo observar que las niñas muestran un desarrollo de sus funciones ejecutivas superior al de los niños en Tercero, Cuarto, Quinto, Sexto, Séptimo básico y en Primero medio $(p s<.01)$. La tendencia opuesta fue observada en Kínder, Primero, Segundo y 
Tabla 5. Medias y Desviación Estándar (DE) en dominios por género y escolaridad.

\begin{tabular}{|c|c|c|c|c|c|c|c|c|c|}
\hline \multirow[t]{2}{*}{ FM } & & \multicolumn{8}{|c|}{$\begin{array}{c}\text { Dominios } \\
\text { Media } \\
\text { (Desviación Estándar) }\end{array}$} \\
\hline & & M & $\mathrm{FE}$ & $\mathrm{P}$ & M & $\mathrm{L}$ & A & HS & PE-C \\
\hline \multirow[t]{3}{*}{ Kínder } & Niño & $\begin{array}{c}0.246 \\
(0.174)\end{array}$ & $\begin{array}{c}0.584 \\
(0.347)\end{array}$ & $\begin{array}{c}0.462 \\
(0.301)\end{array}$ & $\begin{array}{c}0.451 \\
(0.367)\end{array}$ & $\begin{array}{c}0.408 \\
(0.346)\end{array}$ & $\begin{array}{l}-- \\
--\end{array}$ & $\begin{array}{c}0.248 \\
(0.244)\end{array}$ & $\begin{array}{c}0.250 \\
(0.243)\end{array}$ \\
\hline & Niña & $\begin{array}{c}0.339 \\
(0.234)\end{array}$ & $\begin{array}{c}0.666 \\
(0.349)\end{array}$ & $\begin{array}{c}0.457 \\
(0.248)\end{array}$ & $\begin{array}{c}0.330 \\
(0.240)\end{array}$ & $\begin{array}{c}0.235 \\
(0.192)\end{array}$ & -- & $\begin{array}{c}0.211 \\
(0.180)\end{array}$ & $\begin{array}{c}0.244 \\
(0.191)\end{array}$ \\
\hline & Total & $\begin{array}{c}0.282 \\
(0.202)\end{array}$ & $\begin{array}{c}0.618 \\
(0.346)\end{array}$ & $\begin{array}{c}0.460 \\
(0.277)\end{array}$ & $\begin{array}{c}0.402 \\
(0.324)\end{array}$ & $\begin{array}{c}0.332 \\
(0.298)\end{array}$ & & $\begin{array}{c}0.231 \\
(0.215)\end{array}$ & $\begin{array}{l}0.248 \\
(0.220)\end{array}$ \\
\hline \multirow[t]{3}{*}{$1^{\circ} \mathrm{EB}$} & Niño & $\begin{array}{c}0.169 \\
(0.206)\end{array}$ & $\begin{array}{c}0.450 \\
(0.300)\end{array}$ & $\begin{array}{c}0.300 \\
(0.309)\end{array}$ & $\begin{array}{c}0.310 \\
(0.299)\end{array}$ & $\begin{array}{c}0.267 \\
(0.261)\end{array}$ & $\begin{array}{c}0.563 \\
(0.347)\end{array}$ & $\begin{array}{c}0.185 \\
(0.256)\end{array}$ & $\begin{array}{c}0.227 \\
(0.248)\end{array}$ \\
\hline & Niña & $\begin{array}{c}0.302 \\
(0.322)\end{array}$ & $\begin{array}{c}0.674 \\
(0.423)\end{array}$ & $\begin{array}{c}0.373 \\
(0.343)\end{array}$ & $\begin{array}{c}0.467 \\
(0.375)\end{array}$ & $\begin{array}{c}0.340 \\
(0.325)\end{array}$ & $\begin{array}{c}0.419 \\
(0.444)\end{array}$ & $\begin{array}{c}0.233 \\
(0.268)\end{array}$ & $\begin{array}{c}0.260 \\
(0.160)\end{array}$ \\
\hline & Total & $\begin{array}{c}0.229 \\
(0.268)\end{array}$ & $\begin{array}{c}0.554 \\
(0.374)\end{array}$ & $\begin{array}{c}0.329 \\
(0.319)\end{array}$ & $\begin{array}{c}0.381 \\
(0.339)\end{array}$ & $\begin{array}{c}0.301 \\
(0.290)\end{array}$ & $\begin{array}{c}0.515 \\
(0.375)\end{array}$ & $\begin{array}{c}0.204 \\
(0.256)\end{array}$ & $\begin{array}{c}0.242 \\
(0.209)\end{array}$ \\
\hline \multirow[t]{3}{*}{$2^{\circ} \mathrm{EB}$} & Niño & $\begin{array}{c}0.303 \\
(0.230)\end{array}$ & $\begin{array}{c}0.645 \\
(0.338)\end{array}$ & $\begin{array}{c}0.396 \\
(0.302)\end{array}$ & $\begin{array}{c}0.338 \\
(0.367)\end{array}$ & $\begin{array}{c}0.243 \\
(0.240)\end{array}$ & $\begin{array}{c}0.594 \\
(0.377)\end{array}$ & $\begin{array}{c}0.220 \\
(0.231)\end{array}$ & $\begin{array}{c}0.301 \\
(0.370)\end{array}$ \\
\hline & Niña & $\begin{array}{c}0.181 \\
(0.217)\end{array}$ & $\begin{array}{c}0.707 \\
(0.260)\end{array}$ & $\begin{array}{c}0.408 \\
(0.277)\end{array}$ & $\begin{array}{c}0.418 \\
(0.299)\end{array}$ & $\begin{array}{c}0.234 \\
(0.187)\end{array}$ & $\begin{array}{c}0.702 \\
(0.429)\end{array}$ & $\begin{array}{c}0.288 \\
(0.282)\end{array}$ & $\begin{array}{c}0.285 \\
(0.283)\end{array}$ \\
\hline & Total & $\begin{array}{c}0.229 \\
(0.260)\end{array}$ & $\begin{array}{c}0.677 \\
(0.297)\end{array}$ & $\begin{array}{c}0.402 \\
(0.285)\end{array}$ & $\begin{array}{c}0.374 \\
(0.335)\end{array}$ & $\begin{array}{c}0.239 \\
(0.216)\end{array}$ & $\begin{array}{c}0.641 \\
(0.397)\end{array}$ & $\begin{array}{c}0.250 \\
(0.253)\end{array}$ & $\begin{array}{l}0.293 \\
(0.325)\end{array}$ \\
\hline \multirow[t]{3}{*}{$3^{\circ} \mathrm{EB}$} & Niño & $\begin{array}{c}0.514 \\
(0.100)\end{array}$ & $\begin{array}{c}0.944 \\
(0.242)\end{array}$ & $\begin{array}{c}0.488 \\
(0.320)\end{array}$ & $\begin{array}{c}0.650 \\
(0.460)\end{array}$ & $\begin{array}{c}0.746 \\
(0.072)\end{array}$ & $\begin{array}{c}0.896 \\
(0.383)\end{array}$ & $\begin{array}{c}0.370 \\
(0.288)\end{array}$ & $\begin{array}{c}0.373 \\
(0.378)\end{array}$ \\
\hline & Niña & $\begin{array}{c}0.294 \\
(0.301)\end{array}$ & $\begin{array}{c}0.600 \\
(0.515)\end{array}$ & $\begin{array}{c}0.313 \\
(0.252)\end{array}$ & $\begin{array}{c}0.250 \\
(0.266)\end{array}$ & $\begin{array}{c}0.251 \\
(0.232)\end{array}$ & $\begin{array}{c}0.642 \\
(0.399)\end{array}$ & $\begin{array}{c}0.255 \\
(0.240)\end{array}$ & $\begin{array}{c}0.275 \\
(0.251)\end{array}$ \\
\hline & Total & $\begin{array}{c}0.264 \\
(0.243)\end{array}$ & $\begin{array}{c}0.707 \\
(0.468)\end{array}$ & $\begin{array}{c}0.368 \\
(0.277)\end{array}$ & $\begin{array}{c}0.369 \\
(0.371)\end{array}$ & $\begin{array}{c}0.357 \\
(0.294)\end{array}$ & $\begin{array}{c}0.722 \\
(0.400)\end{array}$ & $\begin{array}{c}0.286 \\
(0.249)\end{array}$ & $\begin{array}{c}0.296 \\
(0.269)\end{array}$ \\
\hline \multirow[t]{3}{*}{$4^{\circ} \mathrm{EB}$} & Niño & $\begin{array}{c}0.302 \\
(0.420)\end{array}$ & $\begin{array}{c}0.752 \\
(0.242)\end{array}$ & $\begin{array}{c}0.313 \\
(0.262)\end{array}$ & $\begin{array}{c}0.438 \\
(0.264)\end{array}$ & $\begin{array}{c}0.335 \\
(0.289)\end{array}$ & $\begin{array}{c}0.745 \\
(0.333)\end{array}$ & $\begin{array}{c}0.296 \\
(0.267)\end{array}$ & $\begin{array}{c}0.362 \\
(0.247)\end{array}$ \\
\hline & Niña & $\begin{array}{c}0.258 \\
(0.293)\end{array}$ & $\begin{array}{c}0.509 \\
(0.344)\end{array}$ & $\begin{array}{c}0.367 \\
(0.247)\end{array}$ & $\begin{array}{c}0.287 \\
(0.275)\end{array}$ & $\begin{array}{c}0.227 \\
(0.365)\end{array}$ & $\begin{array}{c}0.496 \\
(0.407)\end{array}$ & $\begin{array}{c}0.270 \\
(0.267)\end{array}$ & $\begin{array}{c}0.358 \\
(0.313)\end{array}$ \\
\hline & Total & $\begin{array}{c}0.282 \\
(0.363)\end{array}$ & $\begin{array}{c}0.640 \\
(0.360)\end{array}$ & $\begin{array}{c}0.337 \\
(0.253)\end{array}$ & $\begin{array}{c}0.370 \\
(0.276)\end{array}$ & $\begin{array}{c}0.291 \\
(0.322)\end{array}$ & $\begin{array}{c}0.655 \\
(0.376)\end{array}$ & $\begin{array}{c}0.286 \\
(0.263)\end{array}$ & $\begin{array}{c}0.360 \\
(0.274)\end{array}$ \\
\hline \multirow[t]{3}{*}{$5^{\circ} \mathrm{EB}$} & Niño & $\begin{array}{c}0.281 \\
(0.369)\end{array}$ & $\begin{array}{c}0.541 \\
(0.460)\end{array}$ & $\begin{array}{c}0.281 \\
(0.272)\end{array}$ & $\begin{array}{c}0.335 \\
(0.316)\end{array}$ & $\begin{array}{c}0.400 \\
(0.403)\end{array}$ & $\begin{array}{c}0.551 \\
(0.494)\end{array}$ & $\begin{array}{c}0.350 \\
(0.290)\end{array}$ & $\begin{array}{c}0.424 \\
(0.309)\end{array}$ \\
\hline & Niña & $\begin{array}{c}0.173 \\
(0.295)\end{array}$ & $\begin{array}{c}0.353 \\
(0.348)\end{array}$ & $\begin{array}{c}0.135 \\
(0.157)\end{array}$ & $\begin{array}{c}0.143 \\
(0.164)\end{array}$ & $\begin{array}{c}0.095 \\
(0.152)\end{array}$ & $\begin{array}{c}0.310 \\
(0.247)\end{array}$ & $\begin{array}{c}0.172 \\
(0.342)\end{array}$ & $\begin{array}{c}0.224 \\
(0.251)\end{array}$ \\
\hline & Total & $\begin{array}{c}0.219 \\
(0.327)\end{array}$ & $\begin{array}{c}0.438 \\
(0.407)\end{array}$ & $\begin{array}{c}0.202 \\
(0.226)\end{array}$ & $\begin{array}{c}0.231 \\
(0.260)\end{array}$ & $\begin{array}{c}0.238 \\
(0.331)\end{array}$ & $\begin{array}{c}0.418 \\
(0.390)\end{array}$ & $\begin{array}{c}0.255 \\
(0.327)\end{array}$ & $\begin{array}{c}0.312 \\
(0.292)\end{array}$ \\
\hline \multirow[t]{3}{*}{$6^{\circ} \mathrm{EB}$} & Niño & $\begin{array}{c}0.172 \\
(0.157)\end{array}$ & $\begin{array}{c}0.808 \\
(0.453)\end{array}$ & $\begin{array}{c}0.205 \\
(0.177)\end{array}$ & $\begin{array}{c}0.333 \\
(0.269)\end{array}$ & $\begin{array}{c}0.342 \\
(0.305)\end{array}$ & $\begin{array}{c}0.733 \\
(0.448)\end{array}$ & $\begin{array}{c}0.246 \\
(0.197)\end{array}$ & $\begin{array}{c}0.268 \\
(0.257)\end{array}$ \\
\hline & Niña & $\begin{array}{c}0.141 \\
(0.214)\end{array}$ & $\begin{array}{c}0.441 \\
(0.431)\end{array}$ & $\begin{array}{c}0.156 \\
(0.216)\end{array}$ & $\begin{array}{c}0.240 \\
(0.289)\end{array}$ & $\begin{array}{c}0.228 \\
(0.320)\end{array}$ & $\begin{array}{c}0.479 \\
(0.396)\end{array}$ & $\begin{array}{c}0.221 \\
(0.302)\end{array}$ & $\begin{array}{c}0.252 \\
(0.287)\end{array}$ \\
\hline & Total & $\begin{array}{c}0.151 \\
(0.197)\end{array}$ & $\begin{array}{c}0.548 \\
(0.465)\end{array}$ & $\begin{array}{c}0.170 \\
(0.204)\end{array}$ & $\begin{array}{c}0.269 \\
(0.283)\end{array}$ & $\begin{array}{c}0.263 \\
(0.316)\end{array}$ & $\begin{array}{c}0.541 \\
(0.419)\end{array}$ & $\begin{array}{c}0.228 \\
(0.272)\end{array}$ & $\begin{array}{c}0.257 \\
(0.276)\end{array}$ \\
\hline
\end{tabular}




\begin{tabular}{|c|c|c|c|c|c|c|c|c|c|}
\hline \multirow[t]{2}{*}{ FM } & & \multicolumn{8}{|c|}{$\begin{array}{c}\text { Dominios } \\
\text { Media } \\
\text { (Desviación Estándar) }\end{array}$} \\
\hline & & $\mathrm{M}$ & FE & $\mathrm{P}$ & M & $\mathrm{L}$ & A & HS & PE-C \\
\hline $7^{\circ} \mathrm{EB}$ & $\begin{array}{l}\text { Niño } \\
\text { Niña } \\
\text { Total }\end{array}$ & $\begin{array}{c}0.333 \\
(0.259) \\
0.162 \\
(0.203) \\
0.232 \\
(0.238)\end{array}$ & $\begin{array}{c}0.764 \\
(0.412) \\
0.347 \\
(0.321) \\
0.518 \\
(0.409)\end{array}$ & $\begin{array}{c}0.405 \\
(0.353) \\
0.106 \\
(0.156) \\
0.236 \\
(0.295)\end{array}$ & $\begin{array}{c}0.434(0 . \\
264) \\
0.214 \\
(0.286) \\
0.300(0 . \\
293)\end{array}$ & $\begin{array}{c}0.486 \\
(0.411) \\
0.210 \\
(0.244) \\
0.328 \\
(0.346)\end{array}$ & $\begin{array}{c}0.581 \\
(0.469) \\
0.263 \\
(0.256) \\
0.397 \\
(0.385)\end{array}$ & $\begin{array}{c}0.319 \\
(0.367) \\
0.259 \\
(0.228) \\
0.282 \\
(0.281)\end{array}$ & $\begin{array}{c}0.381 \\
(0.366) \\
0.361 \\
(0.314) \\
0.370 \\
(0.331)\end{array}$ \\
\hline $8^{\circ} \mathrm{EB}$ & $\begin{array}{l}\text { Niño } \\
\text { Niña } \\
\text { Total }\end{array}$ & $\begin{array}{c}0.117 \\
(0.137) \\
0.199 \\
(0.365) \\
0.178 \\
(0.322)\end{array}$ & $\begin{array}{c}0.302 \\
(0.300) \\
0.578 \\
(0.491) \\
0.511 \\
(0.463)\end{array}$ & $\begin{array}{c}0.104 \\
(0.108) \\
0.230 \\
(0.337) \\
0.195 \\
(0.295)\end{array}$ & $\begin{array}{c}0.207 \\
(0.238) \\
0.339 \\
(0.416) \\
0.309 \\
(0.383)\end{array}$ & $\begin{array}{c}0.136 \\
(0.208) \\
0.240 \\
(0.297) \\
0.215 \\
(0.278)\end{array}$ & $\begin{array}{c}0.335 \\
(0.360) \\
0.593 \\
(0.474) \\
0.526 \\
(0.456)\end{array}$ & $\begin{array}{c}0.111 \\
(0.120) \\
0.288 \\
(0.416) \\
0.242 \\
(0.369)\end{array}$ & $\begin{array}{c}0.113 \\
(0.135) \\
0.421 \\
(0.489) \\
0.333 \\
(0.439)\end{array}$ \\
\hline $1^{\circ} \mathrm{EM}$ & $\begin{array}{l}\text { Niño } \\
\text { Niña } \\
\text { Total }\end{array}$ & $\begin{array}{c}0.117 \\
(0.166) \\
0.109 \\
(0.153) \\
0.111 \\
(0.145)\end{array}$ & $\begin{array}{c}0.340 \\
(0.424) \\
0.257 \\
(0.299) \\
0.275 \\
(0.301)\end{array}$ & $\begin{array}{c}0.055 \\
(0.078) \\
0.190 \\
(0.293) \\
0.160 \\
(0.262)\end{array}$ & $\begin{array}{c}0.090 \\
(0.000) \\
0.142 \\
(0.127) \\
0.131 \\
(0.112)\end{array}$ & $\begin{array}{c}0.047 \\
(0.000) \\
0.071 \\
(0.130) \\
0.068 \\
(0.119)\end{array}$ & $\begin{array}{c}0.655 \\
(0.536) \\
0.354 \\
(0.210) \\
0.421 \\
(0.294)\end{array}$ & $\begin{array}{c}0.203 \\
(0.026) \\
0.098 \\
(0.189) \\
0.125 \\
(0.167)\end{array}$ & $\begin{array}{c}0.136 \\
(0.149) \\
0.060 \\
(0.050) \\
0.079 \\
(0.079)\end{array}$ \\
\hline
\end{tabular}

Nota. FM: Funciones Motoras, FE: Funciones Ejecutivas, P: Percepción, M: Memoria, L: Lenguaje, A: Aprendizaje, HS: Habilidades Sociales, PE-C: Problemas Emocionales-Conductuales.

Octavo básico, en donde los niños muestran un desempeño superior a las niñas $(p s<.05)$.

Para el dominio de Percepción (P), fue detectado un efecto significativo asociado a la escolaridad, $F(9,274)=$ 4.78; $p<.00 ; \eta p 2=.14$. Sin embargo, no fueron detectados efectos significativos asociados al género y tampoco a la interacción entre el género y curso. En este sentido, las comparaciones de medias con la prueba de Bonferroni indicaron que los alumnos de Kínder obtuvieron mayores puntuaciones que los de Quinto, Sexto y Octavo básico $(p s<.001)$. Mientras que los alumnos de Segundo básico obtuvieron mayor puntaje en esta área, que los alumnos de Sexto básico $(p<.05)$.

Finalmente, fue detectado un efecto significativo asociado a la variable género, en los dominios de Aprendizaje (A), $F(1,211)=6.02, p<.01 ; \eta p 2=.03$, y Lenguaje (L), $F(1$, $268)=7.25 ; p<.05 ; \eta p 2=.03$, donde los niños presentan mayores puntuaciones, y por tanto un desempeño más bajo que las niñas.

Con estos resultados fueron establecidos índices de referencia preliminares de las puntuaciones del cuestionario FTF en Chile (Tabla 5), de acuerdo al género, el nivel de escolaridad y la edad de los niños(as). Información que es complementaria a la informada anteriormente por González (2007) para Kínder y Primer año básico.

\section{Comparación de puntuaciones con baremos suecos}

Para establecer la comparación entre los puntajes del FTF y los baremos establecidos con muestras de Suecia (ver Tabla 6), fue usada la prueba $t$ para una muestra, en cada uno de los rangos de edad establecidos por Korkman, et al. (2005).

En la Tabla 6 se observa que los niños chilenos de 5 años de edad presentaron puntuaciones similares a los de Suecia en 12 (66.7\%) de los 18 subdominios considerados, y mostrando significativamente mayores puntuaciones en seis subdominios, lo que da cuenta de mayores dificultades en las áreas de Atención, Hipoactividad, HiperactividadImpulsividad, Percepción Espacial, Percepción Corporal y Problemas Psíquicos Externalizados. En los rangos de 6 a 8 , los niños chilenos solo fueron similares en 2 (11.11\%) de los 18 subdominios considerados, específicamente en el subdominio Funciones Motoras Gruesas y Habilidades de Comunicación, mientras que en los otros 16 subdominios las puntuaciones indican mayores dificultades de desarrollo en los niños chilenos. Para el rango de los 9 a 12 años, los niños chilenos fueron similares en uno (4.54\%) de los 22 subdominios, en el de Funciones Motoras Gruesas, mientras que en los otros 21 subdominios las puntuaciones indican mayores dificultades de desarrollo en los niños chilenos. 
Tabla 6. Comparación de medias en dominios del FTF Chile con Baremos de Suecia, según rangos etarios.

\begin{tabular}{|c|c|c|c|c|c|}
\hline Subdominios & Edades & $\begin{array}{c}\text { Media (DE) } \\
\text { Chile }\end{array}$ & $\begin{array}{c}\text { Media (DE) } \\
\text { Suecia }\end{array}$ & $t_{(\mathrm{gl})}$ & $p$ \\
\hline \multirow{4}{*}{$\begin{array}{l}\text { Funciones motoras } \\
\text { gruesas }\end{array}$} & 5 & $0.22(0.30)$ & $0.16(0.29)$ & $t_{(22)}=.99$ & .33 \\
\hline & $6-8$ & $0.20(0.28)$ & $0.21(0.31)$ & $t_{(89)}=-.33$ & .74 \\
\hline & $9-12$ & $0.19(0.34)$ & $0.18(0.32)$ & $t_{(140)}=.50$ & .61 \\
\hline & $13-15$ & $0.19(0.30)$ & $0.14(0.23)$ & $t_{(58)}=1.34$ & .19 \\
\hline \multirow{4}{*}{$\begin{array}{l}\text { Funciones motoras } \\
\text { finas }\end{array}$} & 5 & $0.33(0.27)$ & $0.27(0.29)$ & $t_{(22)}=1.12$ & .28 \\
\hline & $6-8$ & $0.33(0.27)$ & $0.19(0.28)$ & $t_{(92)}=5.02$ & .00 \\
\hline & $9-12$ & $0.24(0.32)$ & $0.13(0.22)$ & $t_{(139)}=4.11$ & .00 \\
\hline & $13-15$ & $0.16(0.27)$ & $0.10(0.18)$ & $t_{(58)}=1.92$ & .06 \\
\hline \multirow[t]{4}{*}{ Atención } & 5 & $0.63(0.38)$ & $0.35(0.35)$ & $t_{(21)}=3.44$ & .00 \\
\hline & $6-8$ & $0.79(0.79)$ & $0.39(0.43)$ & $t_{(90)}=9.20$ & .00 \\
\hline & $9-12$ & $0.63(0.49)$ & $0.41(0.49)$ & $t_{(141)}=5.36$ & .00 \\
\hline & $13-15$ & $0.58(0.50)$ & $0.38(0.45)$ & $t_{(58)}=3.19$ & .00 \\
\hline \multirow{4}{*}{$\begin{array}{l}\text { Hiperactividad/ } \\
\text { impulsividad }\end{array}$} & 5 & $0.70(0.54)$ & $0.41(0.38)$ & $t_{(22)}=2.54$ & .02 \\
\hline & $6-8$ & $0.63(0.48)$ & $0.39(0.43)$ & $t_{(89)}=4.67$ & .00 \\
\hline & $9-12$ & $0.51(0.50)$ & $0.29(0.40)$ & $t_{(141)}=5.45$ & .00 \\
\hline & $13-15$ & $0.43(0.47)$ & $0.23(0.34)$ & $t_{(58)}=3.25$ & .00 \\
\hline \multirow[t]{4}{*}{ Hipoactividad } & 5 & $0.35(0.36)$ & $0.13(1.23)$ & $t_{(22)}=3.04$ & .01 \\
\hline & $6-8$ & $0.48(0.42)$ & $0.26(0.37)$ & $t_{(92)}=5.20$ & .00 \\
\hline & $9-12$ & $0.48(0.50)$ & $0.31(0.43)$ & $t_{(141)}=4.19$ & .00 \\
\hline & $13-15$ & $0.42(0.48)$ & $0.32(0.46)$ & $t_{(58)}=1.72$ & .09 \\
\hline \multirow{4}{*}{$\begin{array}{l}\text { Planificación/ } \\
\text { Organización }\end{array}$} & 5 & $0.43(0.45)$ & $0.44(0.43)$ & $t_{(23)}=-.10$ & .92 \\
\hline & $6-8$ & $0.52(0.45)$ & $0.41(0.48)$ & $t_{(93)}=2.54$ & .01 \\
\hline & $9-12$ & $0.47(0.42)$ & $0.38(0.51)$ & $t_{(142)}=2.74$ & .01 \\
\hline & $13-15$ & $0.43(0.51)$ & $0.23(0.39)$ & $t_{(59)}=3.03$ & .00 \\
\hline \multirow[t]{4}{*}{ Percepción espacial } & 5 & $0.35(0.31)$ & $0.19(0.41)$ & $t_{(23)}=2.50$ & .02 \\
\hline & $6-8$ & $0.29(0.34)$ & $0.16(0.25)$ & $t_{(90)}=3.78$ & .00 \\
\hline & $9-12$ & $0.17(0.23)$ & $0.11(0.23)$ & $t_{(141)}=3.28$ & .00 \\
\hline & $13-15$ & $0.19(0.30)$ & $0.09(0.21)$ & $t_{(58)}=2.60$ & .01 \\
\hline \multirow[t]{4}{*}{ Percepción temporal } & 5 & $0.86(0.48)$ & $0.82(0.49)$ & $t_{(21)}=.42$ & .68 \\
\hline & $6-8$ & $0.82(0.53)$ & $0.58(0.54)$ & $t_{(91)}=4.34$ & .00 \\
\hline & $9-12$ & $0.36(0.44)$ & $0.20(0.39)$ & $t_{(141)}=4.45$ & .00 \\
\hline & $13-15$ & $0.21(0.39)$ & $0.08(0.22)$ & $t_{(57)}=2.62$ & .01 \\
\hline
\end{tabular}


Continuación Tabla 6

\begin{tabular}{|c|c|c|c|c|c|}
\hline Subdominios & Edades & $\begin{array}{l}\text { Media (DE) } \\
\text { Chile }\end{array}$ & $\begin{array}{c}\text { Media (DE) } \\
\text { Suecia }\end{array}$ & $t_{(\mathrm{gl})}$ & $p$ \\
\hline \multicolumn{6}{|l|}{ Percepción corporal } \\
\hline & 5 & $0.39(0.37)$ & $0.20(0.36)$ & $t_{(23)}=2.50$ & .02 \\
\hline & $6-8$ & $0.33(0.36)$ & $0.18(0.32)$ & $t_{(88)}=3.93$ & .00 \\
\hline & $9-12$ & $0.30(0.35)$ & $0.13(0.27)$ & $t_{(137)}=5.82$ & .00 \\
\hline & $13-15$ & $0.25(0.33)$ & $0.09(0.22)$ & $t_{(57)}=3.73$ & .00 \\
\hline \multirow[t]{4}{*}{ Percepción visual } & 5 & $0.15(0.23)$ & $0.16(0.28)$ & $t_{(22)}=-.16$ & .89 \\
\hline & $6-8$ & $0.17(0.27)$ & $0.05(0.17)$ & $t_{(88)}=4.50$ & .00 \\
\hline & $9-12$ & $0.13(0.24)$ & $0.04(0.16)$ & $t_{(143)}=4.52$ & .00 \\
\hline & $13-15$ & $0.15(0.32)$ & $0.03(0.15)$ & $t_{(59)}=2.82$ & .01 \\
\hline \multirow[t]{4}{*}{ Memoria } & 5 & $0.32(0.25)$ & $0.34(0.33)$ & $t_{(22)}=-.30$ & .77 \\
\hline & $6-8$ & $0.40(0.34)$ & $0.24(0.26)$ & $t_{(91)}=4.68$ & .00 \\
\hline & $9-12$ & $0.28(0.28)$ & $0.20(0.30)$ & $t_{(140)}=3.67$ & .00 \\
\hline & $13-15$ & $0.29(0.34)$ & $0.17(0.25)$ & $t_{(57)}=2.85$ & .01 \\
\hline \multirow{4}{*}{$\begin{array}{l}\text { Comprensión del } \\
\text { lenguaje }\end{array}$} & 5 & $0.41(0.34)$ & $0.33(0.32)$ & $t_{(21)}=1.20$ & .24 \\
\hline & $6-8$ & $0.45(0.38)$ & $0.21(0.34)$ & $t_{(89)}=6.17$ & .00 \\
\hline & $9-12$ & $0.35(0.40)$ & $0.16(0.36)$ & $t_{(139)}=5.74$ & .00 \\
\hline & $13-15$ & $0.34(0.43)$ & $0.12(0.24)$ & $t_{(57)}=3.95$ & .00 \\
\hline \multirow[t]{4}{*}{ Expresión del lenguaje } & 5 & $0.28(0.29)$ & $0.21(0.28)$ & $t_{(20)}=1.18$ & .25 \\
\hline & $6-8$ & $0.27(0.34)$ & $0.09(0.15)$ & $t_{(87)}=5.12$ & .00 \\
\hline & $9-12$ & $0.25(0.30)$ & $0.08(0.19)$ & $t_{(137)}=6.55$ & .00 \\
\hline & $13-15$ & $0.23(0.33)$ & $0.09(0.20)$ & $t_{(53)}=3.22$ & .00 \\
\hline \multirow{4}{*}{$\begin{array}{l}\text { Habilidades de } \\
\text { comunicación }\end{array}$} & 5 & $0.16(0.27)$ & $0.27(0.35)$ & $t_{(23)}=-1.82$ & 08 \\
\hline & $6-8$ & $0.22(0.37)$ & $0.17(0.32)$ & $t_{(92)}=1.34$ & .18 \\
\hline & $9-12$ & $0.25(0.37)$ & $0.16(0.35)$ & $t_{(139)}=3.07$ & .00 \\
\hline & $13-15$ & $0.29(0.47)$ & $0.11(0.27)$ & $t_{(59)}=3.00$ & .00 \\
\hline \multirow[t]{4}{*}{ Lectoescritura } & 5 & --- & --- & --- & --- \\
\hline & $6-8$ & --- & --- & --- & --- \\
\hline & $9-12$ & $0.50(0.46)$ & $0.28(0.44)$ & $t_{(137)}=5.76$ & .00 \\
\hline & $13-15$ & $0.46(0.45)$ & $0.26(0.40)$ & $t_{(58)}=3.44$ & .00 \\
\hline \multirow[t]{4}{*}{ Matemáticas } & 5 & --- & --- & --- & --- \\
\hline & $6-8$ & --- & --- & --- & --- \\
\hline & $9-12$ & $0.55(0.57)$ & $0.25(0.45)$ & $t_{(142)}=6.27$ & .00 \\
\hline & $13-15$ & $0.49(0.58)$ & $0.24(0.43)$ & $t_{(59)}=3.33$ & .00 \\
\hline \multirow{4}{*}{$\begin{array}{l}\text { Adquisición de } \\
\text { conocimientos }\end{array}$} & 5 & --- & --- & --- & --- \\
\hline & $6-8$ & --- & --- & --- & --- \\
\hline & $9-12$ & $0.52(0.34)$ & $0.14(0.32)$ & $t_{(137)}=13.10$ & .00 \\
\hline & $13-15$ & $0.47(0.39)$ & $0.11(0.26)$ & $t_{(59)}=7.15$ & .00 \\
\hline
\end{tabular}


Continuación Tabla 6

\begin{tabular}{|c|c|c|c|c|c|}
\hline Subdominios & Edades & $\begin{array}{c}\text { Media (DE) } \\
\text { Chile }\end{array}$ & $\begin{array}{c}\text { Media (DE) } \\
\text { Suecia }\end{array}$ & $t_{(\mathrm{gl})}$ & $p$ \\
\hline \multirow{4}{*}{$\begin{array}{l}\text { Situaciones de } \\
\text { aprendizaje }\end{array}$} & 5 & --- & --- & --- & --- \\
\hline & $6-8$ & --- & --- & --- & --- \\
\hline & $9-12$ & $0.56(0.50)$ & $0.32(0.39)$ & $t_{(134)}=5.64$ & .00 \\
\hline & $13-15$ & $0.54(0.53)$ & $0.29(0.39)$ & $t_{(52)}=3.43$ & .00 \\
\hline \multirow[t]{4}{*}{ Habilidades sociales } & 5 & $0.21(0.23)$ & $0.15(0.20)$ & $t_{(17)}=1.21$ & .24 \\
\hline & $6-8$ & $0.22(0.23)$ & $0.13(0.24)$ & $t_{(77)}=3.59$ & .00 \\
\hline & $9-12$ & $0.26(0.28)$ & $0.12(0.25)$ & $t_{(126)}=5.60$ & .00 \\
\hline & $13-15$ & $0.24(0.31)$ & $0.12(0.24)$ & $t_{(52)}=2.89$ & .01 \\
\hline \multirow{4}{*}{$\begin{array}{l}\text { Problemas psíquicos } \\
\text { internalizados }\end{array}$} & 5 & $0.13(0.22)$ & $0.10(0.24)$ & $t_{(22)}=.73$ & .47 \\
\hline & $6-8$ & $0.24(0.28)$ & $0.09(0.15)$ & $t_{(87)}=5.15$ & .00 \\
\hline & $9-12$ & $0.27(0.31)$ & $0.12(0.19)$ & $t_{(140)}=6.01$ & .00 \\
\hline & $13-15$ & $0.31(0.39)$ & $0.12(0.24)$ & $t_{(57)}=3.78$ & .00 \\
\hline \multirow{4}{*}{$\begin{array}{l}\text { Problemas psíquicos } \\
\text { externalizados }\end{array}$} & 5 & $0.38(0.32)$ & $0.19(0.25)$ & $t_{(22)}=2.85$ & .01 \\
\hline & $6-8$ & $0.36(0.32)$ & $0.14(0.22)$ & $t_{(89)}=6.44$ & .00 \\
\hline & $9-12$ & $0.40(0.35)$ & $0.13(0.25)$ & $t_{(134)}=9.22$ & .00 \\
\hline & $13-15$ & $0.37(0.43)$ & $0.13(0.25)$ & $t_{(56)}=4.32$ & .00 \\
\hline \multirow{4}{*}{$\begin{array}{l}\text { Obsesivos- } \\
\text { compulsivos }\end{array}$} & 5 & $0.14(0.18)$ & $0.08(0.16)$ & $t_{(22)}=1.58$ & .13 \\
\hline & $6-8$ & $0.17(0.29)$ & $0.09(0.24)$ & $t_{(87)}=2.66$ & .01 \\
\hline & $9-12$ & $0.19(0.27)$ & $0.08(0.21)$ & $t_{(138)}=4.82$ & .00 \\
\hline & $13-15$ & $0.23(0.42)$ & $0.07(0.20)$ & $t_{(56)}=2.98$ & .00 \\
\hline
\end{tabular}

Nota. Los grados de libertad (gl) variaron dependiendo del tramo de edad, porque las muestras tuvieron distintos tamaños. A su vez, para ciertos subdominios no todos los sujetos tenían información, motivo por el cual en un mismo tramo de edad los grados de libertad también variaron.

Finalmente, para el rango de edad comprendido entre los 13 y 15 años, los puntajes fueron similares a los puntajes de los niños de Suecia en tres (13.6\%) de los 22 subdominios, con igualdad en las Funciones Motoras Gruesas; las Funciones Motoras Finas e Hipoactividad. Todo esto indica que a partir de los 6 años de edad, los niños chilenos tienen un perfil en el cual se detectan significativamente más problemas asociados a cada una de las áreas que evalúa el FTF.

\section{Discusión}

En el año 2007 comenzaron a investigarse en Chile las propiedades psicométricas del cuestionario FTF, en muestras de niños de Kínder y Primer año de enseñanza básica (González, 2007), obteniéndose índices confiables y validos, que dan cuenta de la utilidad de este instrumento en el contexto chileno. Una vez cumplida esta fase, fue propuesta como meta analizar las propiedades psicométricas, abarcando el amplio espectro de edad para el cual fue diseñado el cuestionario (de 5 a 15 años), de tal forma de asegurar las condiciones para continuar el estudio en muestras clínicas.

Aquí se han estudiado las propiedades psicométricas del instrumento en una muestra de niños de 5 a 15 años, obteniéndose índices de confiabilidad y validez igualmente adecuados, que posicionan al cuestionario FTF como una herramienta viable para ser utilizada en la evaluación del desarrollo infantil. De esta manera el FTF, considerando el amplio rango de edad al cual está dirigido, favorece la evaluación y el seguimiento de los niños a través de su periodo de desarrollo, además de constituir una herramienta que permite realizar estudios longitudinales que podrían contribuir a aumentar la información epidemiológica disponible en Chile.

La diversidad de áreas que evalúa, tales como Funciones motoras, Funciones ejecutivas, Percepción, Aprendizaje, Memoria, Lenguaje, Habilidades sociales y Problemas 
emocionales-conductuales, permitiría obtener una descripción global del desarrollo del niño desde el punto de vista de los padres o cuidadores, como agentes que aportan información relevante acerca del desarrollo de sus hijos, sus pautas de interacción y el impacto que ciertos trastornos originan en la vida cotidiana.

En relación a la consistencia interna del cuestionario FTF, fueron obtenidos índices similares a los alcanzados en investigaciones anteriores, tanto en Europa como en Chile.

Para la escala en general, se obtuvo un coeficiente Alfa de Cronbach de 0.98, al igual que el alcanzado en la investigación realizada en Chile previamente por González (2007). Esta tendencia se mantiene al analizar los coeficientes obtenidos en cada uno de los dominios del cuestionario, que van desde 0.83 (Percepción y Memoria) a 0.93 (Problemas emocionales conductuales y Aprendizaje), superando levemente los índices obtenidos anteriormente en Chile de 0.77 a 0.92 y .81 a 0.93 , para la muestra de Kínder y Primer año básico, respectivamente (González, 2007). Además, ambas investigaciones coinciden en indicar que los dominios con confiabilidades más bajas son los de Percepción, Aprendizaje y Habilidades motoras, mientras que los que presentan mayores confiabilidades son los dominios de Problemas Emocionales-Conductuales y Aprendizaje.

Al comparar la confiabilidad obtenida en esta investigación con los índices alcanzados en estudios de los países europeos nórdicos por Airaksinen et al. (2004), Kadesjö et al. (2004), Korkman, et al. (2004) y Trillingsgaard et al. (2004), es posible afirmar que si bien en Europa los coeficientes Alfa de Cronbach son levemente superiores, en la presente investigación el cuestionario FTF muestra niveles de confiabilidad que varían de buenos a muy buenos, asegurando consistencia y estabilidad temporal de las mediciones. En este estudio por ejemplo, a diferencia del estudio de González (2007), se analizó la confiabilidad test-retest. Con base a estos resultados fue posible establecer que si bien los índices son levemente inferiores a los obtenidos en la investigación realizada por Kadesjö, et al. (2004), todos los subdominios, a excepción de Hipoactividad y Percepción visual, muestran correlaciones positivas estadísticamente significativas. Estas diferencias pueden ser atribuidas al hecho que en la investigación europea el FTF fue administrado en un periodo de 6 a 8 semanas de diferencia, mientras que en la presente investigación transcurrió un periodo de al menos 9 meses entre ambas aplicaciones. Tomando en cuenta este antecedente, la confiabilidad testretest del cuestionario FTF es más que adecuada, lo que constituye un requerimiento básico para un instrumento que está dirigido a detectar eventuales trastornos asociados al desarrollo, que se caracterizan por ser persistentes (Avaria, 2005; López-Villalobos, Serrano y Delgado, 2004; Moraga, Sahie y Quezada, 2007).

En relación a los factores del instrumento, acá fueron extraídos cuatro componentes, que en conjunto explican un $65.4 \%$ de la varianza. La forma como los subdominios están cargando en los distintos componentes fue diferente a la organización obtenida en los países nórdicos, que dan cuenta de dos factores (Dificultades de aprendizaje y Problemas socioemocionales), como en la investigación realizada por Bohlin y Janols (2004) o siete factores (Habilidades cognitivas; Habilidades motoras y percepción; Emoción; Socialización y conducta; Atención; Habilidades literarias; y Control), como en la investigación llevada a cabo por Bruce et al., (2006). Creemos que estos resultados previos no son comparables con los obtenidos en la presente investigación, ya que en el estudio de Bohlin y Janols la muestra no contemplaba el espectro completo de edad para el cual fue diseñado el cuestionario, mientras que la investigación de Bruce y colegas fue realizada con una muestra clínica. $\mathrm{Si}$ bien la investigación realizada en Chile por González (2007) se focalizó en una muestra de niños de Kínder y Primer año básico, es posible advertir ciertas concordancias en cuanto a las dimensiones obtenidas por nuestro estudio, ya que en ambos predomina un factor general de desarrollo, que comprende todas las áreas evaluadas por el cuestionario.

Lo anterior significa que al trabajar con niños normales, el instrumento evalúa una sola dimensión del desarrollo que condensa gran parte de la variabilidad, en la cual casi todos los dominios están integrados, mientras que cuando se evalúan muestras clínicas de niños con alteraciones en el desarrollo, emergen más dimensiones, consistentes con los síntomas descritos, los cuales se organizan en conglomerados independientes.

$\mathrm{Al}$ analizar el comportamiento de las puntuaciones del FTF, de acuerdo a la variable género, se mantiene la tendencia de investigaciones anteriores, en las cuales las niñas muestran un desempeño superior al de los niños (Airaksinen et al., 2004; Bohlin y Janols, 2004; Bruce et al., 2006; Kadesjö et al., 2004), sin embargo, este efecto solo es estadísticamente significativo en los dominios de Aprendizaje y Lenguaje.

Lo anteriormente expuesto da cuenta de divergencias con las investigaciones anteriores, que sitúan las diferencias en los dominios de Funciones ejecutivas y Habilidades motoras (Kadesjö et al., 2004); Percepción y Habilidades motoras (Bohlin y Janols, 2004); Habilidades motoras y Aprendizaje (Bruce et al., 2006); Habilidades sociales (Airaksinen et al., 2004) y Habilidades sociales y Funciones ejecutivas (González, 2007). Más allá de estas diferencias en términos de dominios, todos los resultados confirman tendencia general de que las niñas tienen menos indicadores de alteraciones del desarrollo que los niños de su misma edad.

En relación al comportamiento de la muestra según la escolaridad, y asumiendo que esta variable puede ser equiparable a la edad dada su alta correlación, es posible establecer ciertas observaciones. Por ejemplo, que existe una concordancia parcial entre los resultados de esta investigación, en la que se encontraron diferencias por edad en los dominios de Funciones ejecutivas y Percepción, con otros estudios que señalan efectos similares en los dominios de 
Habilidades motoras y Percepción (Bohlin y Janols, 2004), Percepción, Memoria y Lenguaje (Airaksinen et al., 2004); y Funciones motoras, Percepción, Memoria, Lenguaje, Funciones ejecutivas y Aprendizaje (González, 2007).

Un hecho importante de mencionar es que las edades tienden a colapsarse en 4 ó 3 conglomerados. Realmente no hay por qué suponer que entre niños de desarrollo típico deberíamos haber encontrado 10 grupos de edad y/o escolaridad claramente diferenciados. Sin embargo, es importante notar que los puntajes, en la mayoría de los casos, tienden a disminuir a partir de los 6 años de edad, cuando los niños ingresan al sistema escolar, y por consiguiente, si el niño tiene una trayectoria de desarrollo típico, es esperable que sus puntajes permanezcan estables a partir de esa edad.

En relación a las comparaciones realizadas entre las puntuaciones del FTF en la muestra chilena y los parámetros establecidos en muestras de Suecia, se observó que los niños chilenos no presentan diferencias significativas con los niños Europeos en la mayoría de los subdominios a los cinco años de edad, sin embargo, a partir del rango de 6 a 8 años en adelante, los niños chilenos tienden a presentar dificultades en casi todas las áreas evaluadas y por tanto a aumentar significativamente las puntuaciones en los subdominios del FTF.

Si bien este efecto podría ser atribuido a un sesgo muestral, la sistematicidad de la tendencia da cuenta de que existen ciertas variables que intervienen en el desarrollo a contar del sexto año de vida, periodo que coincide con el ingreso al sistema escolar en Chile, el que de algún modo parece interferir en el desarrollo óptimo de los niños, en las diversas áreas evaluadas por el cuestionario FTF. De esta manera, es posible atribuir hipotéticamente las diferencias observadas al efecto de la educación formal, que en Chile depende del ingreso económico de la familia.

En Chile existen tres tipos de colegios según su dependencia administrativa: públicos, administrados por los municipios; particulares que reciben subvención estatal, y particulares pagados, financiados por los apoderados. A los primeros asisten los estudiantes de sectores de más bajos recursos, mientras que a los colegios particulares pagados asisten estudiantes pertenecientes a los sectores de más altos ingresos (Cisternas, 2007). Condiciones diferentes se presentan en los países nórdicos como Suecia, ya que en ese contexto, existe un solo sistema de educación, que se caracteriza por ser de alta calidad, integrador, no selectivo y gratuito (Vega, 2005).

Los resultados en Chile de la Prueba SIMCE, muestran una brecha entre los tres tipos de establecimientos, lo que queda de manifiesto al observar que de los 10 establecimientos educacionales con mejores puntuaciones en esta prueba, 9 corresponden a colegios de tipo Particular Pagado (MINEDUC, 2008), dando cuenta finalmente, que la educación de calidad no es transversal en los tres tipos de establecimientos. Si a esto se suma el hecho de que los establecimientos educacionales con menores recursos albergan una mayor cantidad de niños con déficit en su desarrollo cognitivo, emocional y social (Bralic, Seguel y Montenegro, 1987), es posible indicar que el sistema educacional chileno, con su desigualdad en la distribución de recursos, no logra potenciar el desarrollo de todos los alumnos.

En este sentido, es importante plantear que este estudio con el FTF posiblemente captó el funcionamiento con dificultades de los niños con desarrollo típico, que provienen de familias con menores recursos económicos. Porque en esta investigación hubo una sobre-representación de colegios y escuelas municipales con subvención estatal, y ausencia de colegios particulares pagados. De replicar este estudio con niños de desarrollo típico provenientes de colegios particulares pagados, y encontrar diferencias en el mismo sentido que las observadas en esta investigación, la explicación no podría atribuirse al factor socioeconómico y debería buscarse en las prácticas de crianza, que están culturalmente institucionalizadas y son transversales al tipo de colegio.

Finalmente, al estudiar las propiedades psicométricas del cuestionario FTF y determinar su adecuación al contexto chileno, en una muestra de niños normales, se establecen las bases para continuar con esta investigación en al menos dos áreas: el análisis de la validez discriminante del FTF con niños diagnosticados con TDAH u otro trastorno del desarrollo, y la posibilidad de indagar cómo las variables sociales median los resultados obtenidos por los niños.

Considerando que en investigaciones realizadas con muestras clínicas, el cuestionario FTF ha mostrado altos niveles de sensibilidad (93\%) en la detección de TDAH y sus patologías comórbidas (Korkman, et. al, 2004), es recomendable validar este instrumento en una muestra clínica, que permita confirmar el uso de este cuestionario como una herramienta diagnóstica de trastornos del desarrollo. Asociado a esto, habría que explorar la inclusión de otro tipo de procedimiento o técnica de evaluación, que mejore la especificidad del cuestionario. Porque el riesgo conocido es que muchos niños de desarrollo típico son considerados como niños con trastornos del desarrollo, cuando en la práctica no lo son.

Además, dadas las características de segregación y exclusión del sistema educativo chileno, es importante realizar investigaciones para estudiar el comportamiento de las puntuaciones del FTF, tomando en cuenta variables como nivel socioeconómico y la educación de los padres, y otros aspectos que permitan determinar cómo estas variables interfieren en el desarrollo óptimo del niño(a) y, por otro lado, si existen diferencias significativas a la hora de crear las normas definitivas de esta escala.

\section{Referencias}

Airaksinen, E., Mickelsson, K., y Jokela, V. (2004). The occurrence of inattention, hyperactivity, impulsivity and coexisting symptoms in a population study of 471 6-8-year old children based on the FTF (Five to Fifteen) questionnaire. European Child Adolescent Psychiatry, 13, 23-30. 
Amador, J., Forns, M., y Martorell, B. (2001). Síntomas de desatención e hiperactividad-impulsividad: análisis evolutivo y consistencia entre informantes. Anuario de Psicología, 32, 51-66.

Amador, J., Forns, M., Guárdia, J., y Peró, M. (2006). Estructura factorial y datos descriptivos del perfil de atención y del cuestionario TDAH para niños en edad escolar. Psicothema, 18, 696-703.

American Psychiatric Association (2002). Manual diagnóstico y estadístico de los trastornos mentales (DSMIV-TR). Barcelona: Masson.

Avaria, M. (2005). Pediatría del desarrollo y comportamiento. Revista Pediatría Electrónica, 21, 88-91.

Babinsky, L., Hartsough, C., y Lambert, N. (1999). Childhood Conduct Problems, Hyperactivity-impulsivity and Inattention as Predictors of Adult Criminal Activity. Journal of Child Psychology and Psychiatic, 40, 347-355.

Bedregal, P., Scharager, J., Breinbauer, C., Solari, J., y Molina, H. (2007). El desarrollo de un modelo para la evaluación de rezagos del desarrollo infantil de Chile. Revista Médica de Chile, 135, 403-405.

Bedregal, P. (2008). Instrumentos de medición del desarrollo en Chile. Revista Chilena de Pediatría, 79, 32-36.

Belfer, M. (2008). Child and adolescent mental disorders: the magnitude of the Problem across the globe. Journal of Child Psychology and Psychiatry, 49, 226-236.

Bohlin, G., y Janols, L. (2004). Behavioural problems and psychiatric symptoms in 5-13 year-old Swedish children - a comparison of parent ratings on the FTF (Five to Fifteen) with the ratings on CBCL (Child Behavior Checklist). European Child Adolescent Psychiatry, 13, 14-22.

Bralic, S., Seguel, X., y Montenegro, H. (1987). Prevalencia de trastornos psíquicos en la población escolar de Santiago. Disponible en www. cedep.cl.

Bruce, B., Thernlund, G., y Nettelbladt, U. (2006). ADHD and language impairment, A study of the parent questionnaire FTF (Five to Fifteen). European Child Adolescent Psychiatry, 15, 52-60.

Cisternas, F. (2007). Reforma educacional, capital humano y desigualdad en Chile. Horizontes educacionales, 12, 43-53.

Cova, F., Maganto, C., y Melipillán, R. (2005). Adversidad familiar y desarrollo de trastornos internalizados y externalizados en preadolescentes. Revista Chilena de Neuro-Psiquiatría, 43, 287-296.

De la Barra, F., Toledo, V., y Rodríguez, J. (2002). Estudio de salud mental en dos cohortes de niños escolares de Santiago Occidente. I Prevalencia y seguimiento de problemas conductuales y cognitivos. Revista Chilena de Neuro-Psiquiatría, 40, 9-21.

De la Barra, F., Toledo, V., y Rodríguez, J. (2003). Estudio de salud mental en dos cohortes de niños escolares de Santiago Occidente. III Predictores tempranos de problemas conductuales cognitivos. Revista Chilena de Neuro-Psiquiatría, 41, 65-74.

Ferrán, P., Claustre, M., Canals, J., Esparó, G., Ballespi, S., y Doménech, E. (2008). Evaluación de la psicopatología del preescolar mediante el Early ChildhoodInventory-4 (ECI-4): concordancia entre padres y maestros. Psicothema, 20, 481-486.

George, M., Siraquian, X., y Mores, R. (1995). Adaptación y validación de dos instrumentos de pesquisa de problemas de salud mental en escolares de $1^{\circ}$ básico. Revista de Psicología, 5, 17-25.

González, C. (2007). El five to fifteen (FTF): Evaluación del desarrollo de funciones cognitivas y de ajuste socioemocional para pre-escolares y escolares (Memoria de pregrado para optar al título de psicólogo de la Universidad de Talca, Chile. Recuperada de http://dspace.utalca.cl/ handle/1950/6473).

Haeussler, I., y Marchant, T. (2002). Tepsi. Test de desarrollo psicomotor 2-5 años. Santiago: Ediciones Universidad Católica de Chile.

Kadesjö, B., Janols, L., Korkman, M., Mickelsson, K., Strand, G., Trillingsgaard, A., y Gillberg, C. (2004). The FTF (Five to Fifteen): the development of a parent questionnaire for the assessment of ADHD and comorbid conditions. European Child Adolescent Psychiatry, 13, 3-13.

Kordas, K., Stoltzfus, R., López, P., Alatorre, J., y Rosado, J. (2005). Iron and zinc supplementation does not improve parent or teacher ratings of behavior in first grade Mexican children exposed to lead. The Journal of Pediatrics, 147, 632-639.

Korkman, M., Jaakkola, M., Ahlroth, A., Pesonen, A., y Turunen, M. (2004). Screening of developmental disorders in five-year-olds using the FTF (Five to Fifteen) questionnaire: a validation study. European Child Adolescent Psychiatry, 13, 31-38.

Korkman, M., Kadesjö, B., Trillingsgaard, A., Mickelsson, K., Janols, L., Strand, G., y Gillberg, C. (2005). 5-15 (FTF) Parent questionnaire for evaluation of development and behavior of 5 to 15 -year-old children.

Leslie, L., Hurlburt, M., Landsverk, J., Barth, R., y Slymen, D. (2004). Outpatient mental health services for children in foster care: A national perspective. Child Abuse and Neglect, 28, 697-712.

López-Villalobos, J., Serrano, I., y Delgado, J. (2004). Trastorno por déficit de atención con hiperactividad: comorbilidad con trastornos depresivos y de ansiedad. Psicothema, 16, 402-407.

MINEDUC. (2008). Informe de resultados nacionales SIMCE. Disponible en www.simce.cl.

Moraga, F., Sahie, C., y Quezada, A. (2007). Respuestas a la audiencia con el Comité Asesor Presidencial de Políticas para la Infancia. Revista Chilena de Pediatría, 78, 51-56.

Power, T., Doherty, B., Panichelli, S., Karustis, J., Eiraldi, R., Anastopoulos, A., y DuPaul, G. (1998). The predictive validity of parent and teacher report of ADHD symptoms. Journal of Psychopathology and Behavioral Assessment, 20, 57-81.

Rodríguez, S., Arancibia, V., y Undurraga, C. (1976). Escala de evaluación de desarrollo psicomotor para niños entre 0 y 2 años. Santiago: Editorial Galdoc.

Rodríguez, S., Lira, M., Arancibia, V., y Bralic, S. (1996). Inventario de problemas conductuales y socioemocionales: 3-5 años. Santiago: Ediciones Universidad Católica.

Schonhaut, L., Maggiolo, M., De Barbieri, Z., Rojas, P., y Salgado, A (2007). Dificultades de lenguaje en preescolares: Concordancia entre el test TEPSI y la evaluación fonoaudiológica. Revista Chilena de Pediatría. 78, 369-375.

Trillingsgaard, A., Damm, D., Sommer, S., Jepsen, J., Ostergaard, O., Frydenberg, M., y Thomsen, P. (2004). Developmental profiles on the basis of the FTF (Five to Fifteen) questionnaire. Clinical validity and utility of the FTF in a child psychiatric sample. European Child Adolescent Psychiatry, 13, 39-49.

Vega, L. (2005). Los sistemas educativos Europeos y la formación de profesores: Los casos de Francia, Reino Unido, España y Finlandia. Revista de Educación, 336, 169-187.

Wechsler, D. (1999). Escala de inteligencia de Wechsler para niños revisada ( $5^{\mathrm{a}}$ ed.). Madrid: TEA. 
